ARTICLE

\title{
Inhibitors of BRAF dimers using an allosteric site
}

\author{
Xiomaris M. Cotto-Rios (10) 1,2,8, Bogos Agianian (10) 1,2,8凶 , Nadege Gitego ${ }^{1,2}$, Emmanouil Zacharioudakis ${ }^{1,2}$, \\ Orsi Giricz (10 ${ }^{3}$, Yang Wu T,2, Yiyu Zou $^{2,3}$, Amit Verma ${ }^{3,4,5}$, Poulikos I. Poulikakos ${ }^{6,7}$ \& \\ Evripidis Gavathiotis (10) 1,2,5凶
}

BRAF kinase, a critical effector of the ERK signaling pathway, is hyperactivated in many cancers. Oncogenic BRAF ${ }^{V 600 E}$ signals as an active monomer in the absence of active RAS, however, in many tumors BRAF dimers mediate ERK signaling. FDA-approved RAF inhibitors poorly inhibit BRAF dimers, which leads to tumor resistance. We found that Ponatinib, an FDA-approved drug, is an effective inhibitor of BRAF monomers and dimers. Ponatinib binds the BRAF dimer and stabilizes a distinct $\alpha \mathrm{C}$-helix conformation through interaction with a previously unrevealed allosteric site. Using these structural insights, we developed PHI1, a BRAF inhibitor that fully uncovers the allosteric site. PHI1 exhibits discrete cellular selectivity for BRAF dimers, with enhanced inhibition of the second protomer when the first protomer is occupied, comprising a novel class of dimer selective inhibitors. This work shows that Ponatinib and BRAF dimer selective inhibitors will be useful in treating BRAF-dependent tumors.

\footnotetext{
${ }^{1}$ Department of Biochemistry, Albert Einstein College of Medicine, Bronx, NY, USA. ${ }^{2}$ Department of Medicine, Albert Einstein College of Medicine, Bronx, NY, USA. ${ }^{3}$ Department of Oncology, Albert Einstein College of Medicine, Montefiore Medical Center, Bronx, NY, USA. ${ }^{4}$ Department of Developmental \& Molecular Biology, Albert Einstein College of Medicine, Bronx, NY, USA. ${ }^{5}$ Albert Einstein Cancer Center, Albert Einstein College of Medicine, Bronx, NY, USA. ${ }^{6}$ Department of Oncological Sciences, Icahn School of Medicine at Mount Sinai, New York, USA. ${ }^{7}$ Department of Dermatology, Icahn School of Medicine at Mount Sinai, New York, USA. ${ }^{8}$ These authors contributed equally: Xiomaris M. Cotto-Rios, Bogos Agianian. ${ }^{凶}$ email: bogos.agianian@einsteinmed.org; evripidis.gavathiotis@einsteinmed.org
} 
T he RAS-RAF-MEK-ERK signaling pathway (ERK signaling) regulates mammalian cell growth, proliferation, and survival ${ }^{1,2}$. This pathway is normally activated by receptor tyrosine kinase signaling that stimulates binding of GTP to RAS at the plasma membrane $e^{3,4}$. RAF proteins (ARAF, BRAF, and CRAF isoforms) are subsequently recruited at the membrane by interaction with the active GTP-bound RAS, where they are activated by dephosphorylation and phosphorylation events and simultaneous dimerization of their RAF kinase domains $s^{1,2,5}$. Activated RAF proteins subsequently initiate a cascade of phosphorylation and activation steps of downstream kinases MEK1/2 and ERK1/2, which then phosphorylate an array of proteins leading to specific cellular effects $1,2,5$.

Aberrant activation of ERK signaling is a hallmark of many cancers most commonly due to mutations of RAS and BRAF ${ }^{6,7}$. BRAF mutants are found in up to $9 \%$ of all human cancers and over $50 \%$ of melanoma ${ }^{8,9}$. While RAF proteins activate ERK signaling as homo and heterodimers in the presence of active RAS $^{10}$, mutant BRAFV600E can activate ERK signaling independent of RAS as an active monomer ${ }^{11,12}$. Drug development efforts have yielded three FDA-approved RAF inhibitors, Vemurafenib, Dabrafenib, and Encorafenib for $\mathrm{BRAF}^{\mathrm{V} 600 \mathrm{E}}$ metastatic melanoma $a^{13,14}$. These inhibitors show remarkable clinical efficacy in $\mathrm{BRAF}^{\mathrm{V} 600 \mathrm{E}}$ melanoma tumors from potent inhibition of the monomeric $\mathrm{BRAF}^{\mathrm{V} 600 \mathrm{E}}$ protein ${ }^{11-14}$. However, drug resistance to these inhibitors is developed resulting in only short-term improvement of patients' survival ${ }^{15}$. Several mechanisms of clinical resistance to RAF inhibitors have been identified, including feedback reactivation of receptor tyrosine kinases and RAS, RAS mutations, BRAF amplification and expression of $\mathrm{BRAF}^{\mathrm{V} 600 \mathrm{E}}$ splice variants ${ }^{16-19}$. These resistance mechanisms commonly lead to reactivation of ERK signaling through dimerization of RAF proteins ${ }^{16-19}$. RAF dimers are poorly inhibited by the FDA-approved inhibitors ${ }^{11,12}$ and therefore have been recognized as an important target for limiting drug resistance but also for more effective inhibition of ERK signaling in various tumors ${ }^{20,21}$.

Recently, next-generation RAF inhibitors that equipotently inhibit RAF monomers and dimers have been developed ${ }^{11-14}$. Comperative analysis of crystal structures of BRAF kinase bound to RAF inhibitors showed that inhibitors that stabilized the aChelix toward the inactive OUT position ( $\mathrm{aC}$-OUT inhibitors), such as Vemurafenib, Dabrafenib and PLX7094, sterically disfavor binding of the inhibitor to the second protomer within the RAF dimer (negative allostery) ${ }^{12,22,23}$. In contrast, RAF inhibitors that stabilized the $\mathrm{aC}$-helix toward the active IN position ( $\mathrm{aC}$-IN inhibitors), such as TAK-632, LY3009120, and AZ-628, favor catalytic inhibition of both RAF protomers within the dimer by two inhibitor molecules ${ }^{12,24,25}$. Biochemical data consistently demonstrated that $\mathrm{aC}$-IN inhibitors are more effective in inhibiting dimeric RAF activity compared to aC-OUT inhibitors ${ }^{11,12}$. However, aC-IN inhibitors strongly promote RAF binding to active RAS (RAF priming) and therefore induce increased RAF dimerization. In contrast, aC-OUT inhibitors only weakly promote RAF priming ${ }^{12,26}$. Nevertheless, with increased active RAS levels, aC-OUT inhibitors can induce RAF priming and RAF dimerization resulting to the phenomenon described as inhibitorinduced paradoxical activation. This is due to the inability of aCOUT inhibitors to effectively inhibit both protomers, but inhibit only one protomer, within the RAF dimer ${ }^{27-29}$. Therefore, negative allostery and induced RAF priming are two allosteric mechanisms that hamper both aC-OUT and aC-IN RAF inhibitors, underscoring the need for improved rational designed strategies to effectively target BRAF.

Interestingly, although aC-OUT inhibitors show significant selectivity for active $\mathrm{BRAF}^{\mathrm{V} 600 \mathrm{E}}$ monomers compared to BRAF- mutant or wild-type dimers, $\alpha \mathrm{C}$-IN inhibitors inhibit BRAF monomers and dimers or each protomer within a RAF dimer equipotently ${ }^{11,12,26}$. Lack of selectivity by $\mathrm{aC}$-IN inhibitors can significantly reduce their therapeutic index of inhibiting mutant RAF dimers in cancer cells compared to inhibiting wild-type RAF dimers in normal cells ${ }^{12}$. Notably, LY3009120, a potent RAF dimer aC-IN inhibitor, showed limited dose escalation and at its maximum tolerated dose demonstrated limited efficacy in recent phase I trial ${ }^{30}$. Thus, we hypothesized that selective RAF dimer inhibitors may be superior for inhibition of resistant RAF dimers in tumors. Here, we screened a panel of kinase inhibitors with diverse structures in a cell-based assay that probed the activity of obligated $\mathrm{BRAF}^{\mathrm{V} 600 \mathrm{E}}$ dimers ${ }^{19}$. We found that the FDA-approved drug Ponatinib is a potent BRAF inhibitor. Ponatinib inhibited ERK signaling activity in cancer cells driven by either BRAF monomers or dimers. Structural analysis demonstrated that Ponatinib, in contrast to other RAF inhibitors, binds BRAF forming interactions with an allosteric site. Using these structural insights, we developed Ponatinib Hybrid Inhibitor 1 (PHI1) that displays cellular selectivity for $\mathrm{BRAF}^{\mathrm{V} 600 \mathrm{E}}$ and non-V600E dimers over monomers via enhanced inhibition of the second protomer within the dimers, when the first protomer is occupied. Structural investigation revealed that PHII recognizes a previously uncharacterized allosteric site in RAF kinases, which is intimately linked to the conformation of aC-helix. Our studies present mechanistic insights and BRAF inhibitors that can be exploited for the treatment of BRAF dimer-dependent tumors.

\section{Results}

Screening for RAF dimer kinase inhibitors. To identify RAF dimer kinase inhibitors, we established an in-cell-western based screening assay using SKMEL239-C4 melanoma cells ${ }^{31}$. These cells were generated under Vemurafenib-induced selection pressure inhibiting $\mathrm{BRAF}^{\mathrm{V} 600 \mathrm{E}}$, allowing preferential growth of cells expressing p61BRAF ${ }^{\mathrm{V} 600 \mathrm{E}}$, a splice variant of BRAF that constitutively signals as a dimer in a RAS-independent manner ${ }^{19}$ (Fig. 1a). p61BRAF ${ }^{\mathrm{V} 600 \mathrm{E}}$ is resistant to Vemurafenib and is found in patients' tumors ${ }^{19}$. Typically, Vemurafenib robustly inhibits ERK signaling in $\mathrm{BRAF}^{\mathrm{V} 600 \mathrm{E}}$ expressing cells such as melanoma A375 cells at $0.3 \mu \mathrm{M}$, whereas in SKMEL239-C4 cells a similar effect required over $10 \mu \mathrm{M}$ (Supplementary Fig. 1a, b) ${ }^{11,12}$. The in-cell-western assay, which was based on fluorescence readout for phosphorylated ERK (p-ERK) and total ERK levels, enabled a wide dynamic range of signal detection and recapitulated p-ERK resistance of SKMEL239-C4 cells at low concentrations of Vemurafenib (Supplementary Fig. 1c, d). In contrast, Trametinib, an FDA-approved MEK inhibitor, potently inhibited ERK signaling at low nM concentrations (Supplementary Fig. 1e). We used a 96-well format with non-inhibitory concentrations of Vemurafenib as negative control and $0.1 \mu \mathrm{M}$ Trametinib as positive control, obtaining a Z-factor of 0.68 (Supplementary Fig. 1f).

We screened a library of 200 kinase inhibitors, including established RAF and MEK inhibitors as positive controls, and found several additional compounds with capacity to reduce $\mathrm{p}$ ERK levels by more than $50 \%$ at $5 \mu \mathrm{M}$ after $3 \mathrm{~h}$ of treatment (Fig. 1b, c). SKMEL239-C4 cells were co-treated with $0.5 \mu \mathrm{M}$ Vemurafenib in order to saturate inhibition of low expressing $\mathrm{BRAF}^{\mathrm{V} 600 \mathrm{E}}$ monomer and reveal inhibition of p61BRAF $\mathrm{V}^{\mathrm{V}} 00 \mathrm{E}$ dimer ${ }^{19}$. Hits were screened again with or without Vemurafenib co-treatment as control. Several kinase inhibitors were confirmed to have potent inhibitory effect on p-ERK, independently of Vemurafenib (Supplementary Fig. 2). Since p61BRAFV600E maintains activity in SKMEL239-C4 cells independently of active RAS or tyrosine kinase receptor signaling, our screening assay 
a

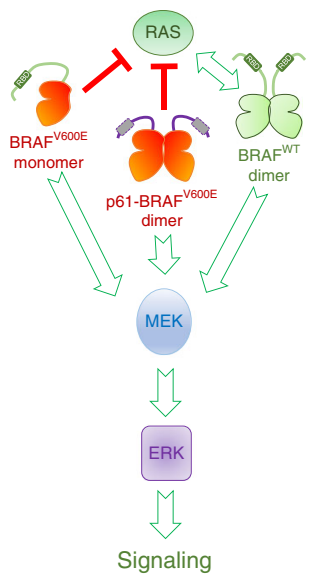

b

C
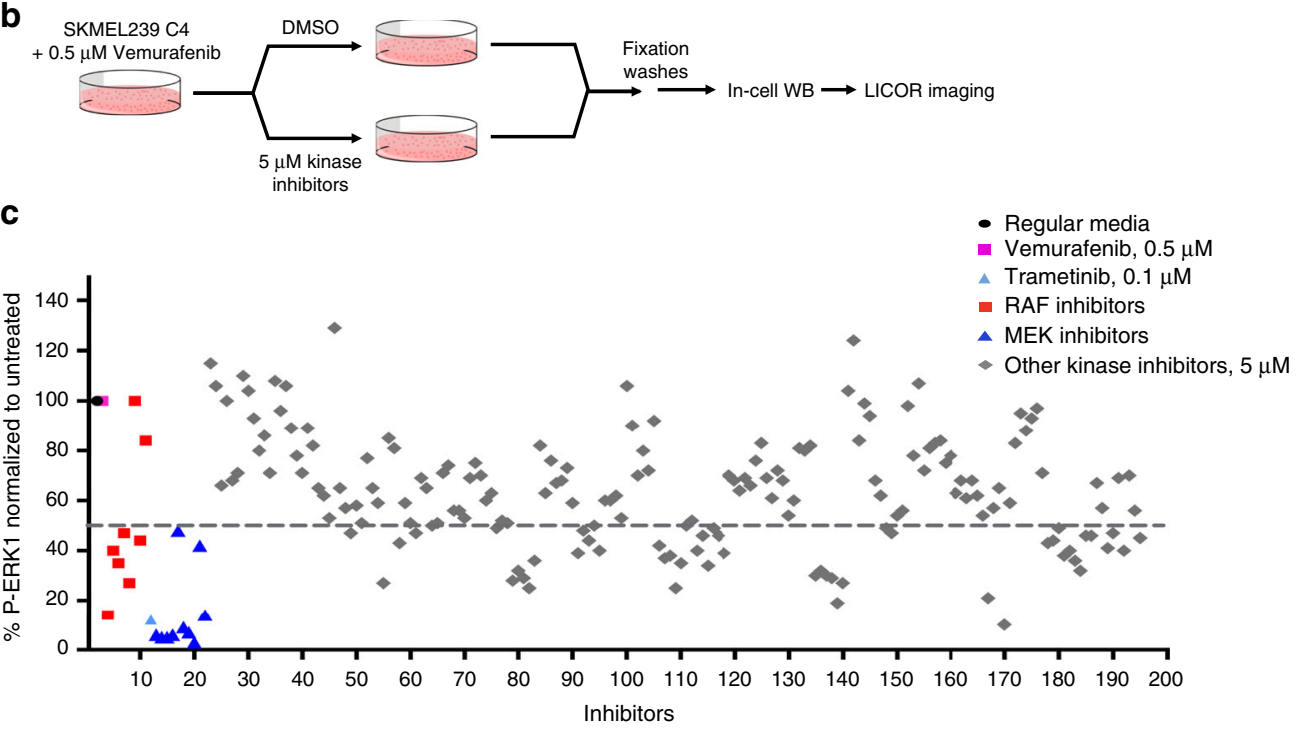

d

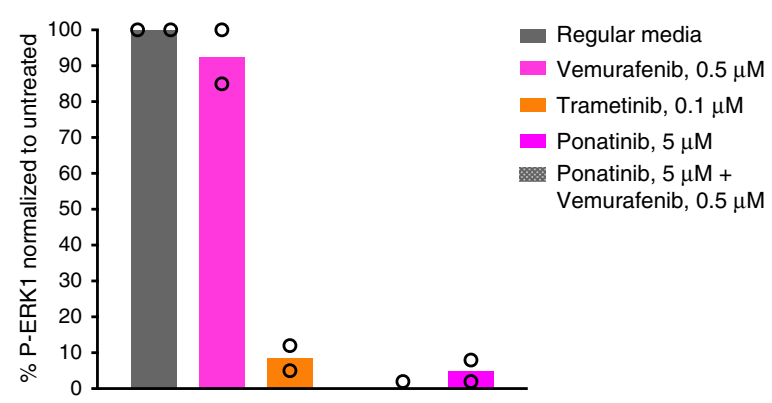

e

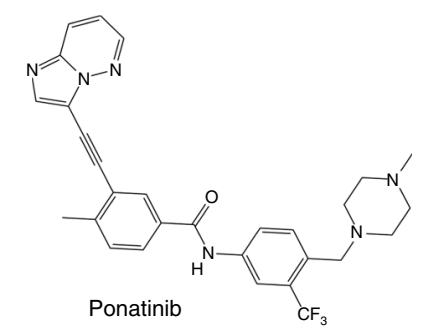

f

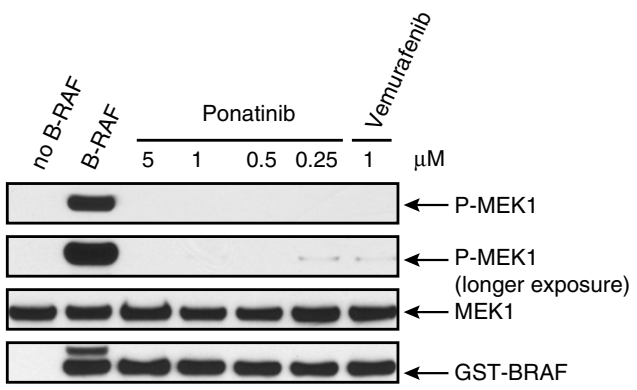

g

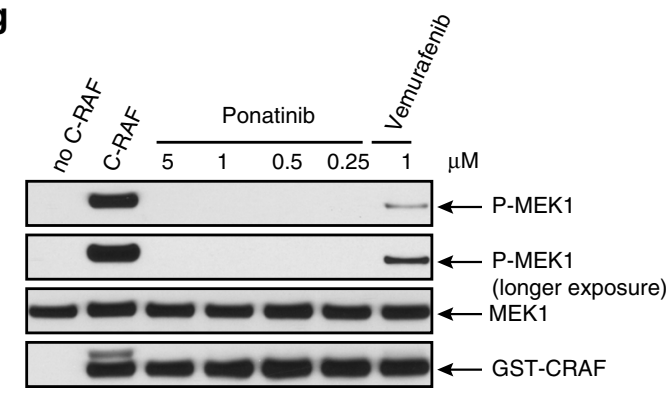

BRAF $^{\text {WT }}$

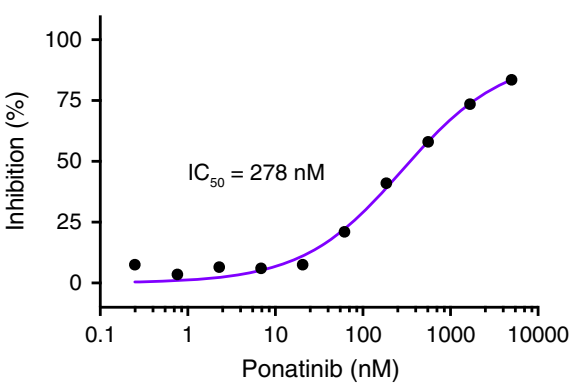

ABL-T315I mutant ${ }^{32,33}$. Besides ABL in CML, Ponatinib strongly inhibits other kinases such as FGFR, FLT3, KIT, and PDFGRa

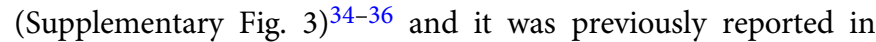
kinome screens to inhibit BRAF activity, however, it is not validated or recognized as BRAF inhibitor ${ }^{13,37}$. We performed in vitro kinase assays for $\mathrm{BRAF}^{\mathrm{V} 600 \mathrm{E}}$ activity to phosphorylate recombinant MEK and for MEK1 activity to phosphorylate
Ponatinib is a potent BRAF inhibitor. Following validation of hits, we focused on Ponatinib, which is an FDA-approved kinase inhibitor for chronic myeloid leukemia (CML) targeting BCR- identified potential BRAF dimer inhibitors ${ }^{12,19}$ (Supplementary Fig. 2). Ponatinib induced one of the strongest inhibitory effect on p-ERK (Fig. 1d, e). 
Fig. 1 Ponatinib is a RAF inhibitor. a Schematic representation of ERK signaling pathway from different BRAF species. $\mathbf{b}$ Schematic representation of the incell-western screening assay. c Screening of a library of 200 kinase inhibitors using in-cell-western assay. SKMEL239-C4 melanoma cells left untreated (regular media), treated with $0.5 \mu \mathrm{M}$ Vemurafenib, $0.1 \mu \mathrm{M}$ Trametinib, or with $5 \mu \mathrm{M}$ of known RAF, MEK, and other kinase inhibitors in the presence of 0.5 $\mu \mathrm{M}$ Vemurafenib for $3 \mathrm{~h}$. d SKMEL239-C4 melanoma cells left untreated (regular media), treated with $0.5 \mu \mathrm{M}$ Vemurafenib, $0.1 \mu \mathrm{M}$ Trametinib, Ponatinib $5 \mu \mathrm{M}$ without or with $0.5 \mu \mathrm{M}$ Vemurafenib for $3 \mathrm{~h}$, and assayed with in-cell-western. Data are mean of $n=2$ independent experiments. e Chemical structure of Ponatinib. $\mathbf{f}$ BRAF kinase activity assay in the absence or presence of Ponatinib or Vemurafenib was assayed by western blot with the indicated antibodies. $\mathbf{g}$ CRAF kinase activity assay in the absence or presence of Ponatinib or Vemurafenib was assayed by western blot with the indicated antibodies. $\mathbf{g}$ Kinase activity inhibition profiles of BRAFV600E and BRAFWT upon Ponatinib titration using SelectScreen assay. Data are mean of two technical replicates from $n=2$ independent experiments. Source data are provided as a Source Data file.

recombinant ERK2, respectively. We found that Ponatinib is a potent inhibitor of MEK phosphorylation by $\mathrm{BRAF}^{\mathrm{V} 600 \mathrm{E}}$, but does not inhibit MEK (Fig. If and Supplementary Fig. 4). In addition, Ponatinib potently inhibited CRAF (Fig. 1g). Ponatinib inhibited BRAFV600E $\left(\mathrm{IC}_{50}=14 \mathrm{nM}\right)$ and BRAFWT $\left(\mathrm{IC}_{50}=278\right.$ $\mathrm{nM}$ ) comparably to Vemurafenib, Dabrafenib, and Encorafenib inhibitors but also to LY3009120, AZ-628, and TAK-632 that inhibit both monomers and dimers (Fig. 1h and Supplementary Fig. 5) ${ }^{11,12}$. Thus, our data demonstrate that Ponatinib is an inhibitor of BRAF with similar potency to other RAF inhibitors.

Ponatinib binds BRAF with a unique binding mode. To gain further insights into the mechanism of BRAF inhibition by Ponatinib, we determined the BRAFV600E_Ponatinib co-crystal structure at $2.1 \AA$ resolution (Supplementary Table S1). In this complex, Ponatinib is well-defined in electron density and binds deeply into the BRAF inter-lobe active site cleft (Fig. 2a, b). Ponatinib induces the DFG-OUT conformation and forms an extended network of hydrophobic and polar contacts, reflecting its nanomolar activity (Fig. 2c, d). Its pyridazine group establishes hydrogen bonds to hinge residues and the methyl and trifluoromethyl moieties occupy the hydrophobic and type-II pockets, respectively. The amide linker forms additional hydrogen bonds to the catalytic E501 residue, which however maintains the catalytically important salt bridge with K483 (Fig. 2d) ${ }^{9}$, a signature of active BRAF conformation. Interestingly, the activation loop of the kinase domain is partially disordered (Fig. 2a) ${ }^{12,38}$, whereas, its experimentally determined portion adopts an orientation that docks residue E600 to a unique position compared to other inhibitor-bound $\mathrm{BRAF}^{\mathrm{V} 600 \mathrm{E}}$ structures (Supplementary Fig. 6), most likely a result of Ponatinib binding mode.

Further analysis revealed that Ponatinib adopts a unique recognition mode for a RAF inhibitor. In this binding mode, apart from the previously defined "adenine", "hydrophobic", and "type-II" pockets (Fig. 2c, d), Ponatinib forms extensive interactions with an allosteric site (Fig. 2c, d) defined as backpocket IV (BP-IV) according to Kinase-Ligand Interaction Fingerprints and Structures (KLIFS) database ${ }^{39}$. Indeed, a comparison of Ponatinib binding mode to known BRAF inhibitors illustrates distinct recognition of this allosteric site by the methyl-piperazine moiety of Ponatinib (Fig. 2e, f). These interactions include a bifurcated hydrogen bond with the backbone carbonyl oxygen atoms of $\alpha \mathrm{E}-\beta 6$ loop residues $\mathrm{I} 573$ and H574 and hydrophobic contacts with R575 (Fig. 2d). Interestingly, H574 and R575 belong to the conserved HRD motif that is essential for substrate phosphorylation ${ }^{40}$ (Fig. 2d). R575 recognizes regulatory phosphorylated residues of the activation loop and the highly conserved H574 is part of the kinase regulatory spine (R-spine) ${ }^{40}$. Although Ponatinib binds Receptor Tyrosine Kinases (RTKs) in a similar manner (see below), among RAF inhibitors, HRD recognition is achieved only by Ponatinib. Taken together, our structural analysis suggests that
Ponatinib binding to BRAF involves interactions with a previously unreaveled allosteric site.

Ponatinib induces BRAF dimers with aC-CENTER conformation. Crystal structures of BRAF with inhibitors typically are determined in dimeric conformation and each protomer's $\mathrm{aC}$ helix can adopt different conformations between the IN (active) and OUT (inactive) position. aC-IN inhibitors occupy both protomers within the dimer, which both have an aC-IN conformation. In contrast, aC-OUT inhibitors occupy the first protomer with the aC-OUT conformation and induce the second protomer with an aC-IN conformation, therefore inhibiting the binding of a second aC-OUT inhibitor. Differences in aC conformation between dimers result in asymmetry as shown by the superposition of protomers, which is more pronounced with aCOUT inhibitors (VEM, DAB) and less with aC-IN inhibitors (LY3009120, AZ-628, and TAK-632) ${ }^{12}$ (Supplementary Fig. 7).

The BRAF-Ponatinib structure corroborates the dimeric conformation of the kinase induced by aC-IN inhibitors, with both protomers occupied by Ponatinib (Fig. 3a). Interestingly, Ponatinib induces an intermediate aC-CENTRE position in both protomers (Fig. 3b and Supplementary Fig. 8a), which compose a perfectly symmetric dimer (crystallographic dimer). To further investigate whether the BRAF-Ponatinib complex has a distinct conformation among other Ponatinib-kinase complexes, we compared the orientation of aC-helix in this complex to crystal structures of Ponatinib with ABL, ABL-T315I, FGFR1, FGFR4, and cKIT kinases ${ }^{35-37}$ (Supplementary Fig. 8b). Comparison shows that only the $\alpha \mathrm{C}$-helix in the BRAF-Ponatinib adopts the aC-CENTER position, despite the similar binding mode of Ponatinib in all active sites (Supplementary Fig. 8c).

To determine whether Ponatinib also induces BRAF dimers in solution, we performed size-exclusion chromatography analysis of recombinant BRAF kinase in complex with Ponatinib and compared its migration to apo-BRAF kinase and BRAF kinase complexed to ATP. As shown in Supplementary Fig. 9, stoichiometric amounts of Ponatinib promote formation of BRAF dimers, in contrast to ATP, which produces BRAF monomers. Next, using co-immunoprecipitation assays, we evaluated whether Ponatinib can promote dimerization of endogenous BRAF with CRAF in cell lines expressing BRAF variants such as BRAFV600E, p61BRAFV600E, and BRAFWT/NRAS mutant. Consistent with our in vitro data, Ponatinib-promoted formation of $\mathrm{BRAF}^{\mathrm{V} 600 \mathrm{E}}$, p61BRAFV600E, and BRAFWT dimers with CRAF in all cell lines in a dose-dependent manner (Fig. 3c). Moreover, coimmunoprecipitation assays revealed that Ponatinib enhanced the formation of RAF/MEK complexes in all cell lines in a dosedependent manner, suggesting that Ponatinib-induced BRAF dimers have a conformation compatible for stable interaction with $\mathrm{MEK}^{41}$ (Fig. 3d). Taken together, our data suggest that Ponatinib binds either BRAFV600E monomers or p61BRAFV600E and BRAFWT dimers and promotes and stabilizes BRAF dimers and RAF/MEK complexes. 
a

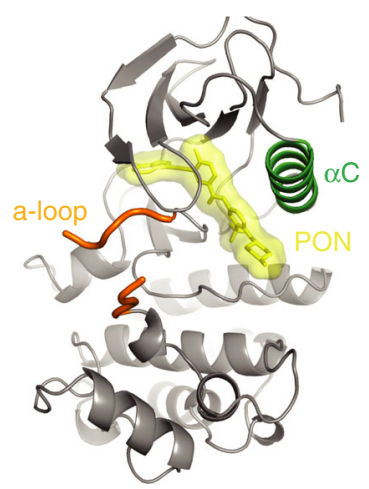

b

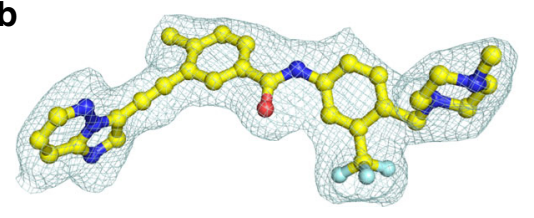

e

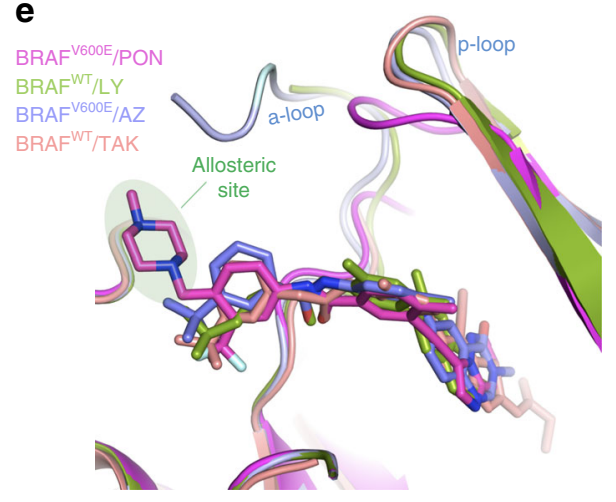

C

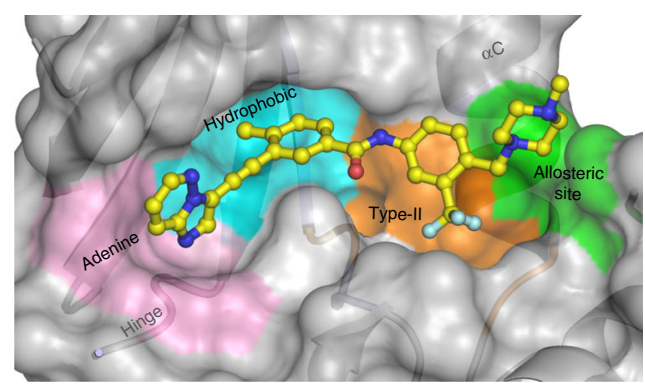

d

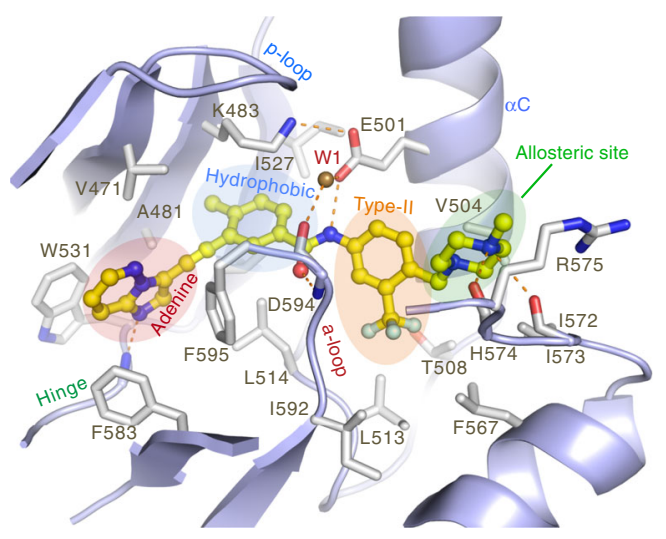

f

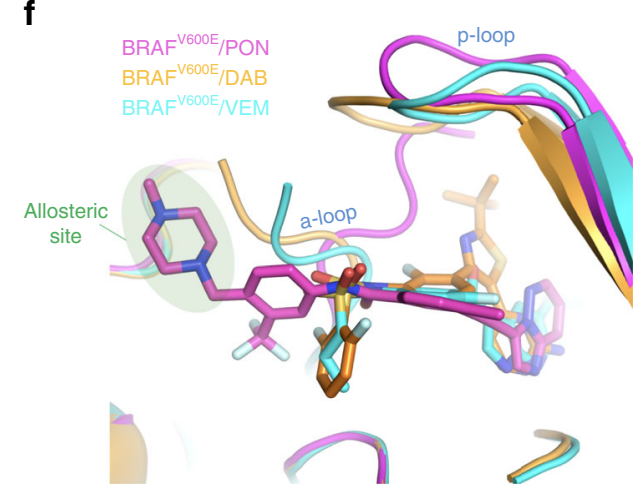

Fig. 2 Structural analysis of BRAFV600E bound to Ponatinib (PON). a Crystal structure of the BRAFV600E/PON protomer structure. BRAFV600E structure depicted in ribbons (gray) showing the $\alpha$ C-helix (green). The partially disordered activation loop (orange) is shown in a tube representation. PON is shown in sticks and molecular surface (yellow). $\mathbf{b} F_{\mathrm{o}}-F_{\mathrm{c}}$ electron density of PON in the BRAFV600E/PON complex contoured at $3 \sigma$. c Close-up of PON binding (in ball-and-sticks) to its binding pocket (transparent surface) in BRAFV600E. Protein sub-pockets recognizing various inhibitor moieties are colored, including the allosteric pocket (green). d A close-up view of PON binding interactions with BRAFV600E. PON is shown in ball-and-sticks and BRAF $V 600 E$ in a cartoon model. Protein residues interacting with PON are shown in sticks. Note hydrogen bond interactions of PON with the backbone of residues $\mathrm{H} 574$ and 1573 in the allosteric site and interaction with the D594 and F595 of the DFG motif and catalytic E501 of the $\alpha \mathrm{C}$-helix. A structural water molecule in the binding site (W1) is shown as a sphere. H-bonds are shown in dashed lines. e A superposition of BRAF type-II/ $\alpha \mathrm{C}-\mathrm{IN}$ inhibitors LY3009120 (LY, green, PDB 5C9C), TAK-632 (TAK, orange, PDB 4KSP), and AZ-628 (AZ, blue, PDB 4G9R) bound in their BRAF recognition pockets with PON in $\mathrm{BRAF}^{\mathrm{V} 600 \mathrm{E}} / \mathrm{PON}$ structure. Protein parts were omitted from clarity. $\mathbf{f} A$ superposition of BRAF type-lb/ $\alpha \mathrm{C}-\mathrm{OUT}$ inhibitors Vemurafenib (VEM, cyan, PDB $30 G 7$ ) and Dabrafenib (DAB, orange, PDB 4XV2) bound in their BRAF recognition pockets with PON in BRAFV600E/PON structure. Inhibitors are depicted in sticks and proteins in a cartoon model.

Ponatinib effectively inhibits oncogenic BRAF. Next, we sought to examine the capacity of Ponatinib to inhibit ERK signaling in tumor cells dependent on BRAFV600E, p61BRAFV600E and $\mathrm{RAS}^{\mathrm{MUT}} / \mathrm{BRAFWT}$. For comparison with a potent inhibitor of

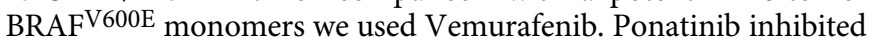
ERK signaling in BRAFV600E and p61BRAFV600E dependent melanoma cells at $0.3-0.5 \mu \mathrm{M}$ doses. However, it required $1-3 \mu \mathrm{M}$ dose to effectively inhibit signaling in $\mathrm{RAS}^{\mathrm{MUT}} / \mathrm{BRAF}^{\mathrm{WT}}$ melanoma or lung cancer cells, suggesting some resistance due to RAF priming similarly to other $\mathrm{aC}^{\mathrm{IN}}$ inhibitors ${ }^{11,12}$ (Fig. 4a). In contrast, Vemurafenib inhibited only $\mathrm{BRAF}^{\mathrm{V} 600 \mathrm{E}}$ monomer expressing cells (A375) but lacks significant inhibitory activity of p-ERK in cells dependent on p61BRAFV600E dimers and enhances p-ERK levels in RASMUT/BRAFWT cells, due to significant negative allostery ${ }^{11-13}$ (Fig. 4a).

To further confirm the direct activity of Ponatinib on BRAF in inhibiting ERK signaling against its potential activity on upstream RTK targets, we used a model system that allows us to assess ERK 
a

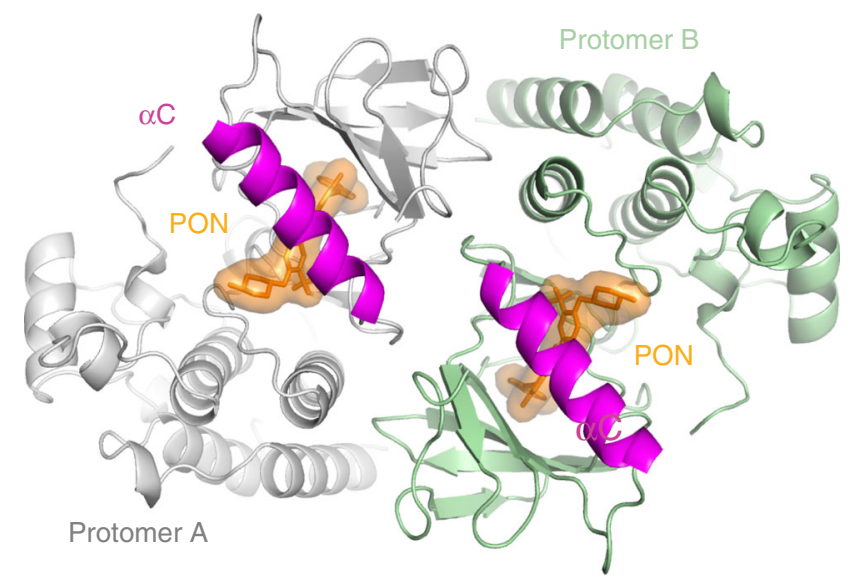

b

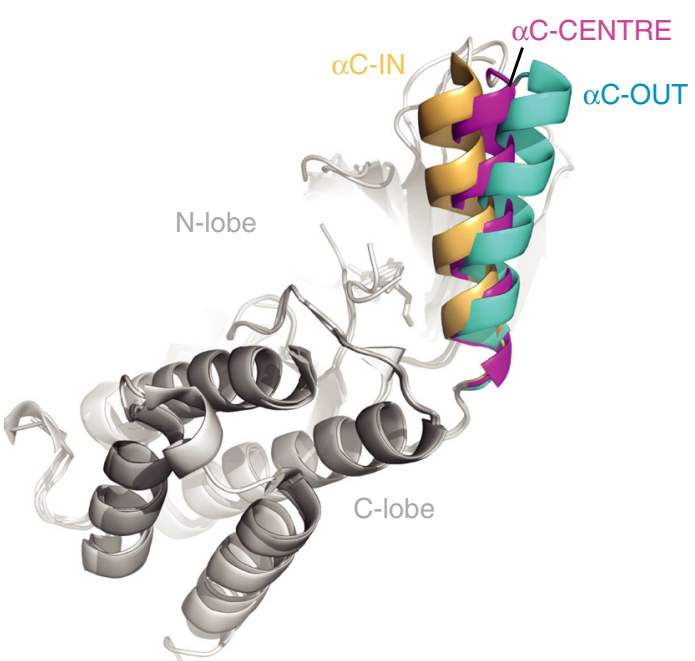

C

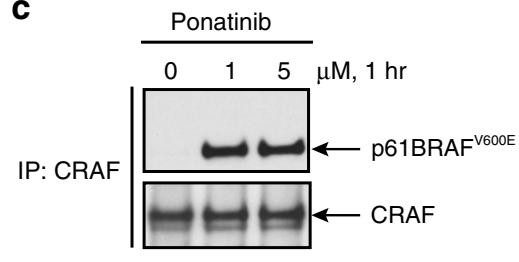

$10 \%$ input

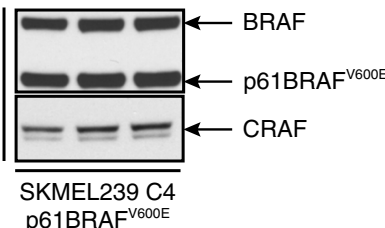

d

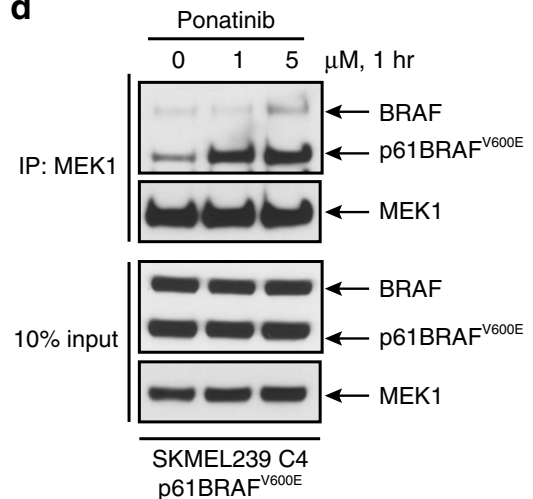

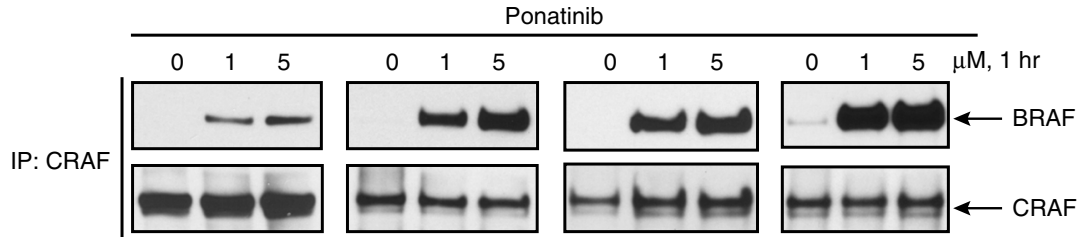
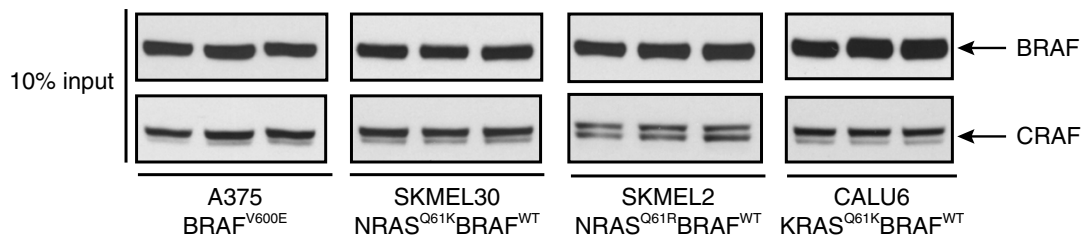

Ponatinib
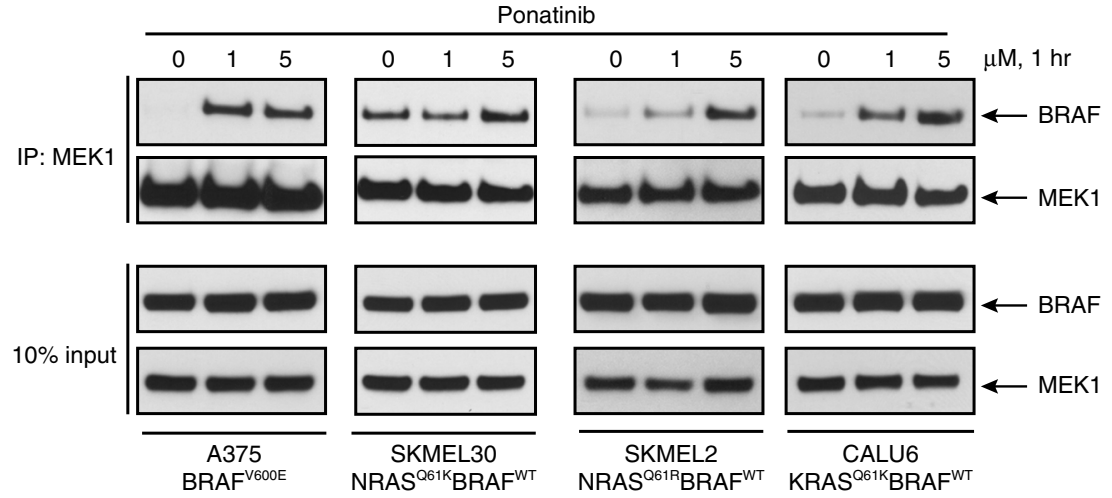

Fig. 3 Ponatinib induces symmetrical BRAF dimers and promotes BRAF complexes in cells. a The BRAFV600E/PON symmetrical dimer structure. The dimer is viewed along its twofold axis with two protomers in silver and green ribbon representations. PON bound to each protomer is shown in orange sticks/molecular surface. The $\alpha \mathrm{C}$-helix of each protomer is illustrated in magenta. $\mathbf{b}$ The $\alpha \mathrm{C}$-helix of BRAFV600E/PON complex adopts a $\alpha \mathrm{C}$-CENTRE position. Structural superposition of BRAF protomer structures in ribbon representation showing that $\alpha \mathrm{C}$-helix of BRAF-PON structure (magenta) lies between typical $\alpha$ C-OUT position observed in BRAF-Vemurafenib complex (cyan, PDB 3OG7) and $\alpha$ C-IN position in MEK-bound BRAF (gold, PDB 4MNE). Protein parts were omitted for clarity. $\mathbf{c}$ Various cell lines expressing BRAFV600E or RASMUT/BRAFWT were left untreated or treated with 1 or $5 \mu \mathrm{M}$ Ponatinib for $1 \mathrm{~h}$. Cells were then collected, assayed for CRAF by immunoprecipitation and immunoblot with the indicated antibodies for monitoring BRAF/ CRAF heterodimerization and activation of ERK signaling. $\mathbf{d}$ Similar analysis to $\mathbf{c}$ assayed for MEK by immunoprecipitation and immunoblot with the indicated antibodies for monitoring BRAF/MEK complex formation. Data are representative of $n=3$ independent experiments. Source data are provided as a Source Data file. 
a
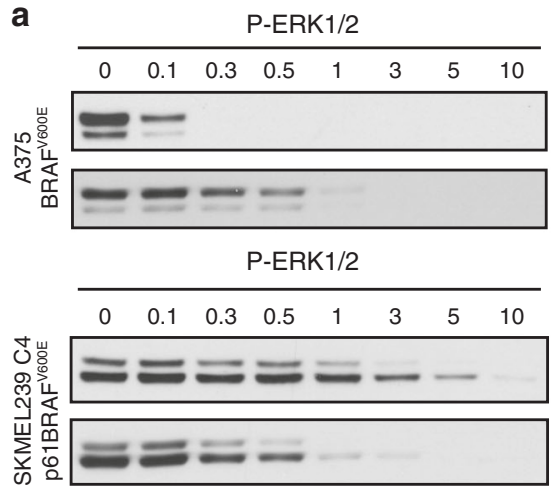

P-ERK1/2
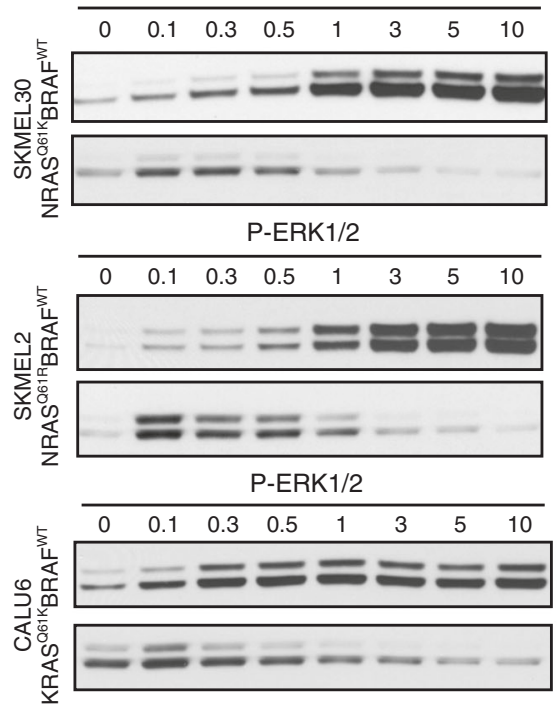

P-MEK1/2
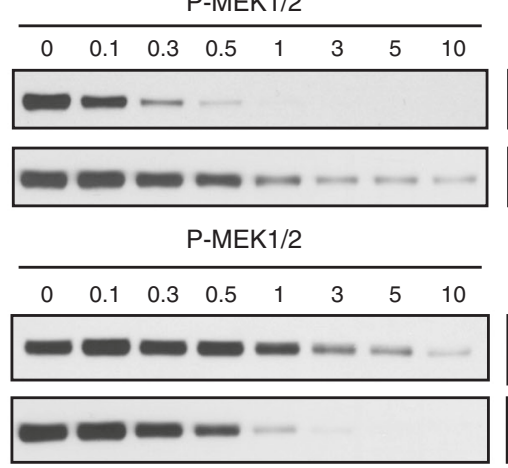

P-MEK1/2

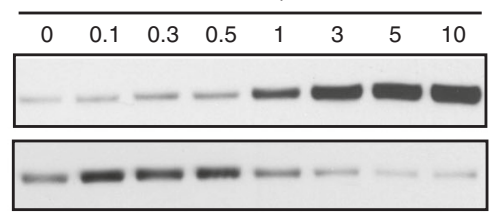

P-MEK 1/2
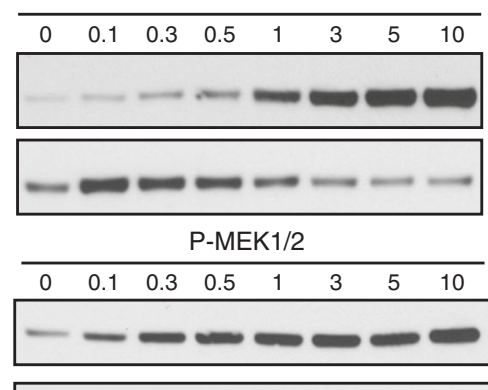

$-\infty-\infty-\cdots$
ERK $1 / 2$

\begin{tabular}{llllllll}
\hline 0 & 0.1 & 0.3 & 0.5 & 1 & 3 & 5 & 10
\end{tabular}$\mu \mathrm{M}, 1 \mathrm{hr}$

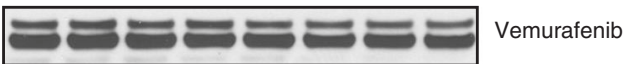

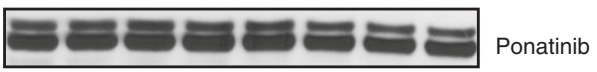

ERK 1/2

\begin{tabular}{lllllllll}
\hline 0 & 0.1 & 0.3 & 0.5 & 1 & 3 & 5 & 10 & $\mu \mathrm{M}, 1 \mathrm{hr}$
\end{tabular}

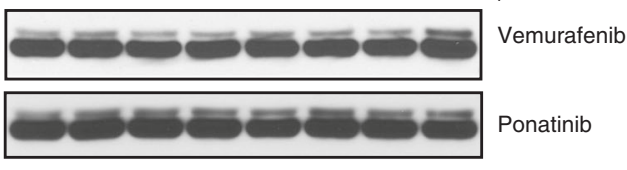

ERK $1 / 2$
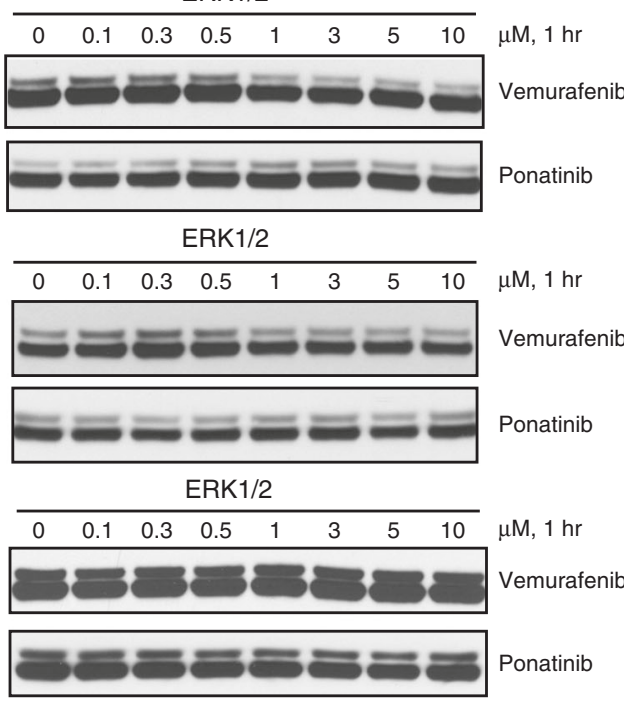

\section{b}

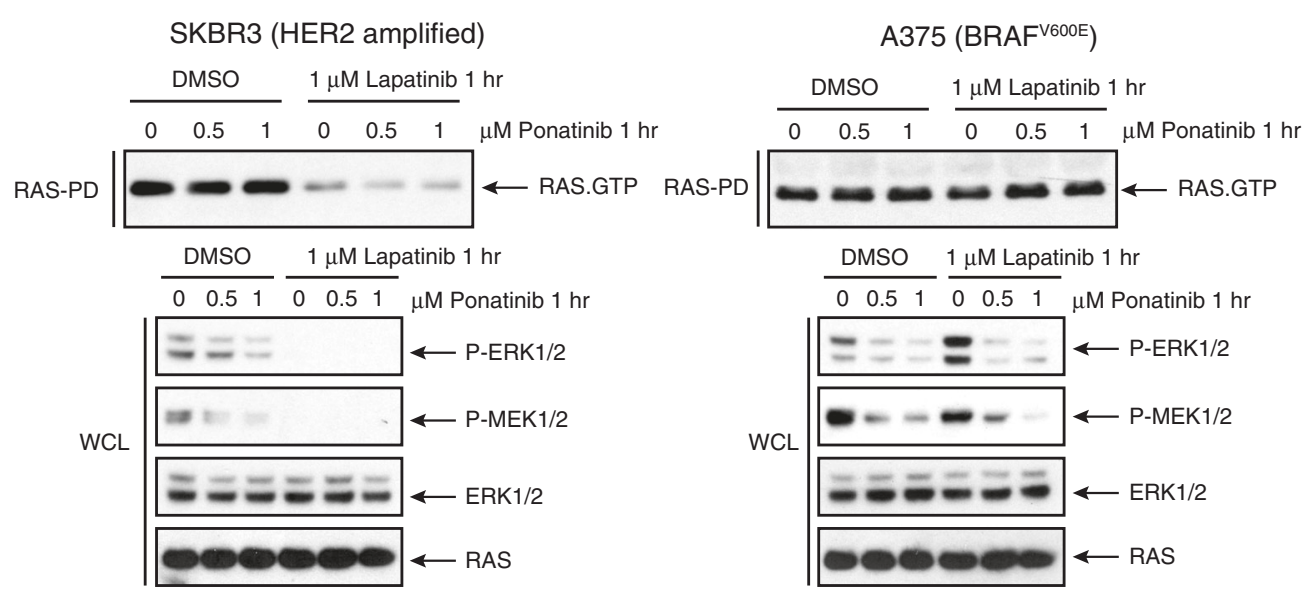

Fig. 4 Ponatinib inhibits different BRAF species and ERK signaling in cells. a Melanoma A375, SKMEL239-C4, SKMEL-30, SKMEL-2, and lung cancer CALU6 cell lines expressing BRAFV600 or RASMUT/BRAFWT left untreated or treated with increasing concentration of Ponatinib or Vemurafenib for $1 \mathrm{~h}$, then assayed for western blot and immunoblot with the indicated antibodies to probe ERK-signaling inhibition. Data are representative of three independent experiments. b Breast cancer SKBR3 cells, which are RTK-dependent HER2 amplified and melanoma A375 (BRAFV600E monomer) cells were left untreated or treated with increasing concentration of Ponatinib and Lapatinib, an EGFR/HER2 inhibitor, for $1 \mathrm{~h}$, then assayed by immunoblot with the indicated antibodies. Data are representative of $n=3$ independent experiments. Source data are provided as a Source Data file.

signaling by RTK inhibition in the absence of active BRAF or RAS-GTP mutations. Thus, we used SKBR3 breast cancer cell line, in which HER2-activated RASWT-GTP levels are attenuated by Lapatinib (dual EGFR/HER2 tyrosine kinase inhibitor) treatment ${ }^{11}$. Ponatinib inhibited ERK signaling without reducing RAS $^{W T}$ _GST levels in SKBR3 cells, whereas Lapatinib inhibited
ERK signaling and significantly reduced RASWT_GST levels, as expected (Fig. 4b). To test whether Ponatinib affects RASWT_GTP levels in an RTK-independent manner, we used A375 cells. Both Ponatinib and Lapatinib did not reduce RASWT_GTP levels, however Ponatinib only inhibited ERK signaling suggesting direct inhibition of BRAF ${ }^{\mathrm{V} 600 \mathrm{E}}$ (Fig. 4b). We also confirmed BRAF 
inhibition by Ponatinib in HEK293 cells whereby ERK signaling was dependent on heterologous expressed p61BRAFV600E (Supplementary Fig. 10). These results suggest that Ponatinib targets BRAF irrespective of RTK targets.

Lastly, we tested the efficacy of Ponatinib to inhibit tumor growth in melanoma cells dependent on BRAFV600E, p61BRAFV600E, and RAS ${ }^{\mathrm{MUT}} / \mathrm{BRAF}^{\mathrm{WT}}$ grown in $3 \mathrm{D}$ culture conditions as previously reported ${ }^{42}$. Ponatinib demonstrated increased efficacy to inhibit colony formation as compared to Vemurafenib in tumor cells expressing BRAFV600E monomers, BRAFV600E $^{\mathrm{V}}$ dimers and RASMUT/BRAFWT dimers (Supplementary Fig. 11). Taken together, these data suggest that Ponatinib is an effective BRAF inhibitor that inhibits melanoma cells dependent on BRAFV600E monomers and dimers as well as mutant RAS-activated BRAFWT dimers with less potency.

Exploiting the BRAF allosteric site with a designed inhibitor. Based on the structural insights of the Ponatib-BRAFV600E dimer structure, we aimed to further exploit the identified BP-IV allosteric site. We performed a computational structure-based drug design approach to replace the head group of Ponatinib structure with alternative fragments that keep the interactions of the trifluoro-phenyl and piperazine interactions of Ponatinib while extending the compound deeper in the allosteric site (Supplementary Fig. 12a, b). Candidate compounds were evaluated in silico for the interactions with the site and filtered for favorable properties compared to Ponatinib (Supplementary Fig. 12a, b and see "Methods" section). Using this approach, we generated PHI1, which uses a morpholine-based head group predicted to interact proximal to the BP-IV site (Supplementary Fig. 12c, d and Supplementary Methods). Indeed, PHI1 showed high inhibitory potency $\mathrm{IC}_{50}=(10 \mathrm{nM})$ against $\mathrm{BRAF}^{\mathrm{V} 600 \mathrm{E}}$ kinase activity (Supplementary Fig. 13).

To investigate the structural basis of BRAF inhibition by PHI, we determined the co-crystal structure of PHI1 with BRAFV600E at $2.65 \AA$ resolution (Supplementary Table S1). Comparison of BRAFV600E_Ponatinib and BRAFV600E_PHI1 structures demonstrated a major allosteric rearrangement induced by PHI1 binding promoting an inward shift of aC-helix (Supplementary Fig. 14). This PHI1-induced BRAF conformational change is compatible with kinase domain dimerization, as a BRAF dimer with two PHI1bound protomers is found in the $\mathrm{BRAF}^{\mathrm{V} 600 \mathrm{E}_{-} \mathrm{PHI} 1}$ crystal structure. Stabilization of BRAF kinase dimer by PHIl is also observed in solution (Supplementary Fig. 9). Interestingly, this PHI1-dependent allosteric modulation stabilizes the aC-helix in a tilted aC-CENTER conformation, distinct from $\alpha \mathrm{C}-\mathrm{IN}$ or $\mathrm{aC}-\mathrm{OUT}$ conformations but closer to aC-IN, which we termed $\alpha \mathrm{C}-\mathrm{IN}^{*}(\alpha \mathrm{C}$ INstar) (Supplementary Fig. 15). This structural change is the result of positioning of the morpholino-based head group of PHI1 in a previously unrevealed extended allosteric BRAF site, which we named aC-allosteric site (Fig. 5a-d). Ponatinib uses its methylpiperazine head group to interact with the HRD motif (Fig. 5a). In contrast, PHI1 positions the morpholino-based head group between the HRD motif and aC-helix, having van der Waals contacts with HRD residue R575 and residues N500 and V504 of aC-helix that result in additional $38 \mathrm{~A}^{2}$ of buried solvent-accessible surface area (Fig. 5b-d). In addition, the specific hydrogen bond between the nitrogen of ethyl-amino linker of PHI1 and the main-chain carbonyl oxygen of H574 is maintained (Fig. 5b-d). Interestingly, comparison between the binding modes of kinase inhibitors across the RAF kinase family using the KLIFS database ${ }^{38}$ suggested that this pocket is distinct from previously reported back- or allosteric pockets (BP-IV or BP-V) and would classify as BP-VI category (Fig. 5e). Notably, this pocket is not predicted in a recent atlas of potential allosteric sites across the kinome ${ }^{43}$.
To evaluate whether the interactions of PHI1 with the BRAF aC-allosteric site can confer selectivity to BRAF compared to other kinases, we compared Ponatinib and PHI1 against a panel of human kinases included in the KinomeEDGE screen (Fig. $5 \mathrm{f}$ and Supplementary Table S2). PHI1 demonstrated a significant gain in overall specificity compared to Ponatinib determined by the Specificity Index S(10) (Fig. 5f), which is comparable to several selective clinical kinase inhibitors ${ }^{44}$. To further validate the results of the kinome screen, we tested PHI1 against established targets of Ponatinib using the SelectScreen assay. Consistently, PHI1 had reduced inhibitory activity in several targets, with FGFR (43-fold reduction) and FLT3 (tenfold reduction) having the highest differences (Fig. 5g). Taken together, our results demonstrated that specific structural changes to BRAF conformation and interactions of PHI1 with the aCallosteric site resulted in increased specificity of PHI1 for BRAF against other kinases.

PHI1 is selective for oncogenic BRAF dimers. Guided by the distinct binding properties and structural effects of PHI1 compared to Ponatinib, we investigated the cellular activity of PHI1. We assessed inhibition activity on BRAF and ERK signaling in melanoma cells expressing BRAFV600E monomers (A375 cells) and constitutively expressed p61BRAFV600E dimers (SKMEL239C4 cells). The inhibitory activity of PHI1 in A375 cells $\left(\mathrm{IC}_{50}=\right.$ $2760 \mathrm{nM})$ is significantly reduced compared to Ponatinib $\left(\mathrm{IC}_{50}=\right.$ $291 \mathrm{nM})$, whereas in SKMEL239-C4 $\left(\mathrm{IC}_{50}=424 \mathrm{nM}\right)$ it is assessed at similar levels compared to Ponatinib $\left(\mathrm{IC}_{50}=452 \mathrm{nM}\right)$ (Fig. 6a, b). Similar selectivity profiles for Ponatinib $\left(\mathrm{IC}_{50}=\right.$ $569 \mathrm{nM}$ ) and PHI1 ( $\mathrm{IC}_{50}=2045 \mathrm{nM}$ ) were also observed in SKMEL239 parental cells expressing BRAFV600E monomers (Supplementary Fig. 16). Consistently, in HEK293 cells ectopically expressing p61BRAFV600E dimers versus p61BRAFV600E harboring the monomer-driver R509H mutation, PHI1 showed preferential inhibition for p61BRAFV600E compared to p61BRAFV600E/R509H BRAF species (Supplementary Fig. 17). Taken together, these results demonstrate that PHI1 more effectively targets p61BRAFV600E dimers than $\mathrm{BRAF}^{\mathrm{V} 600 \mathrm{E}}$ monomers.

These data suggest that the interactions of the morpholinebased head group of PHI1 with the aC-allosteric site controls the specificity for the BRAF dimer. To confirm this, we investigated the activity of PHI2, a compound with an altered head group, but with the same PHI1 and Ponatinib scaffold (Supplementary Fig. 18a). We selected to maintain this common, type-II scaffold, to ensure that the $\mathrm{aC}-\mathrm{IN}$ binding mode to BRAF is maintained. PHI2 potently inhibited BRAF kinase activity in vitro (Supplementary Fig. 18b). However, the inhibitory activity of PHI2 for p61BRAFV600E dimers (SKMEL239-C4 cells) is reduced, while it is increased for BRAFV600E monomers (A375 cells), in comparison to the activity of PHI1 (Supplementary Fig. 18c). Collectively, our data suggest that recognition of the aC-allosteric site by BRAF inhibitors can result in distinct specificity for BRAFV600E dimers.

Since PHI1 is a weak inhibitor of BRAFV600E monomers but inhibits BRAFV600E dimers more effectively, we reasoned that it may be more potent for the second site than the first site within the BRAF dimer. To assess this, we used a previously established assay and treated SKMEL239-C4 cells with $1 \mu \mathrm{M}$ Encorafenib, a potent inhibitor of BRAF ${ }^{\mathrm{V} 600 \mathrm{E}}$ monomers that remains bound to the first site within the dimer up to $24 \mathrm{~h}$ after a wash-out treatment, due to its low $K_{\text {off }}{ }^{11,45}$. Indeed, in control experiments, we observed that Encorafenib treatment for $1 \mathrm{~h}$ inhibited p-ERK, but the activation is recovered after wash-out for another hour due to half-occupied active dimers (Supplementary Fig. 19). 
a

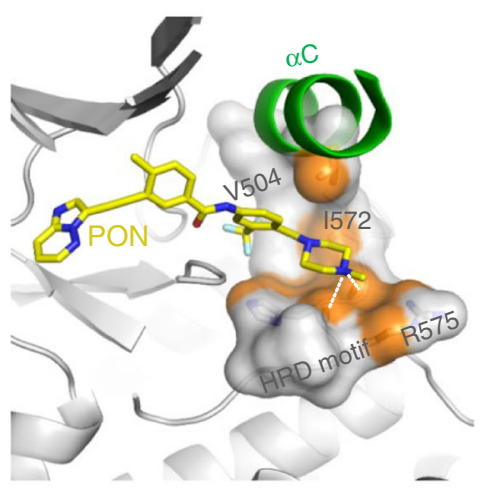

$b$

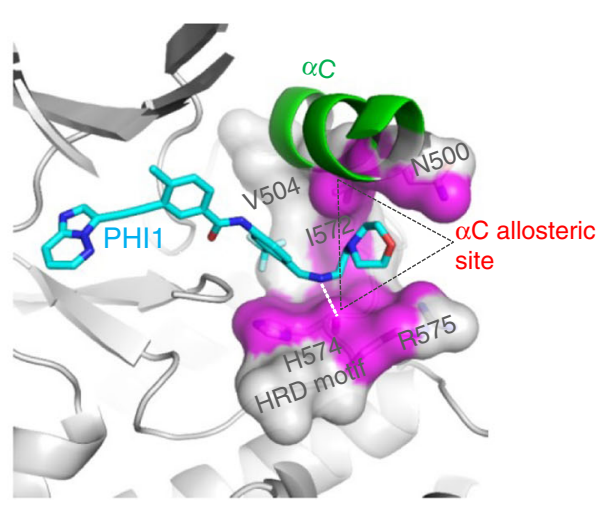

C

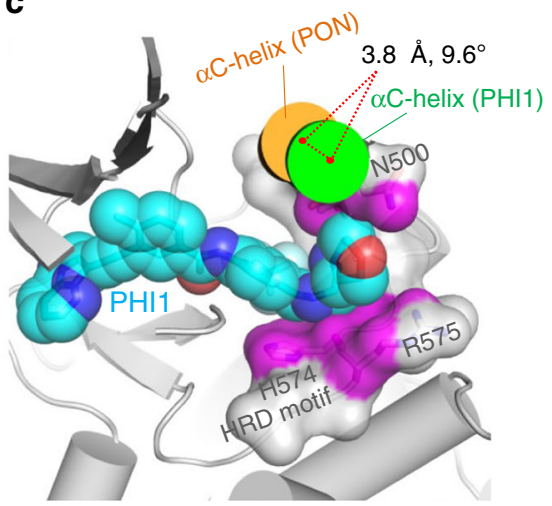

d<smiles></smiles>

$\left(\begin{array}{cc}6 \\ 593\end{array}\right.$

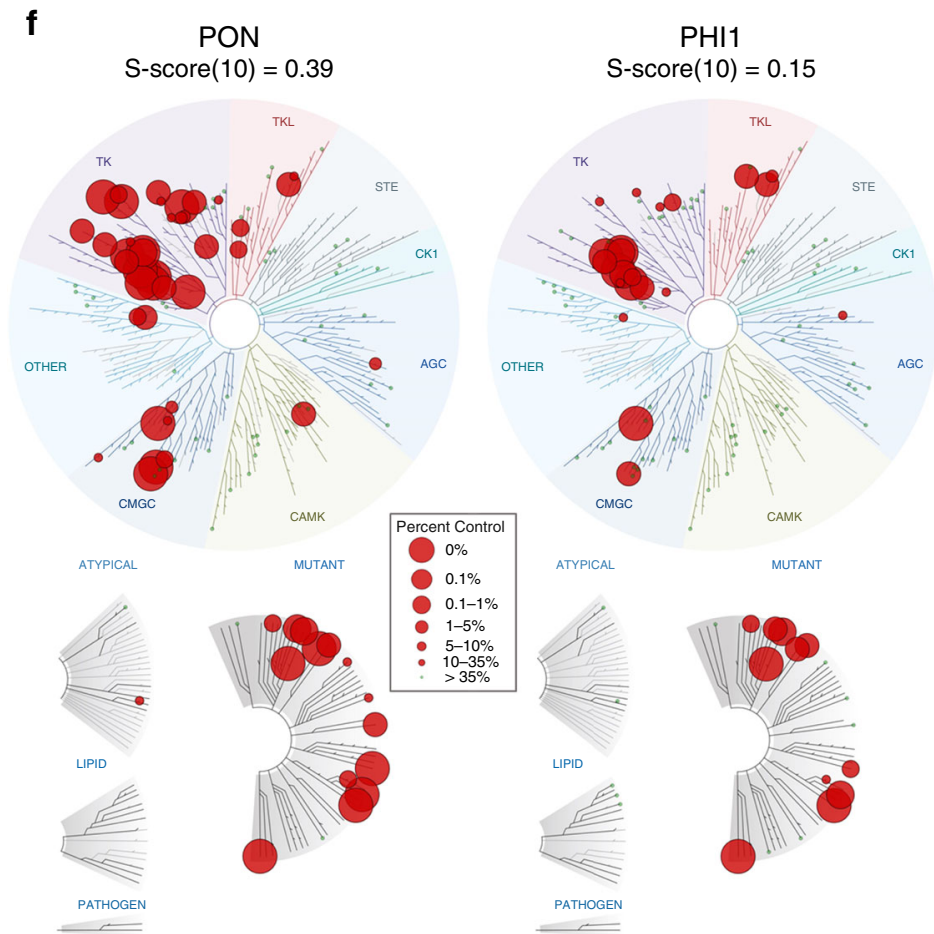

e

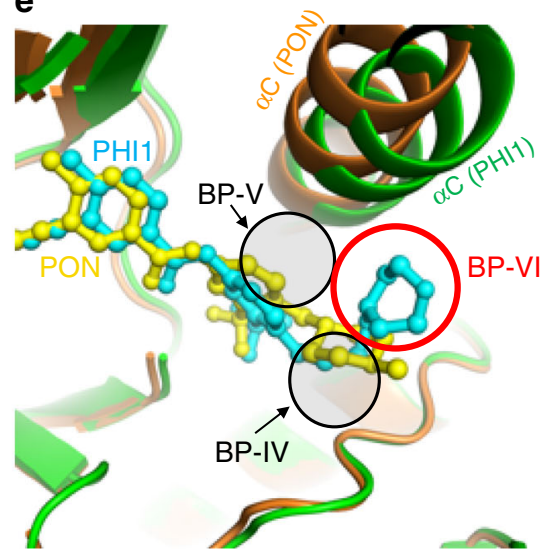

g

\begin{tabular}{|ccc|}
\hline & $\begin{array}{c}\text { PON } \\
\mathrm{IC}_{50}(\mathrm{nM})\end{array}$ & $\begin{array}{c}\text { PHI1 } \\
\mathrm{IC}_{50}(\mathrm{nM})\end{array}$ \\
\hline BRAF & & $10 \pm 2$ \\
\hline BRAFWT & $278 \pm 67$ & $323 \pm 35$ \\
\hline ABL1 & $5.4 \pm 0.4$ & $8.4 \pm 0.3$ \\
\hline FGFR1 & $14.1 \pm 0.7$ & $624 \pm 25$ \\
\hline FLT3 & $2.0 \pm 0.1$ & $2.05 \pm 2.7$ \\
\hline KIT & $483 \pm 39$ & $548 \pm 21$ \\
\hline PDFGR $\alpha$ & $8.0 \pm 1.3$ & $8.3 \pm 1.5$ \\
\hline
\end{tabular}

Following this approach, we determined the inhibitory activity for the second site of the BRAFV600E dimer for various BRAF inhibitors (Fig. 6c-k).

Remarkably, PHI1 inhibited more potently the second site within the BRAFV600E dimer $\left(\mathrm{IC}_{50}=134 \mathrm{nM}\right)$ than the Encorafenib-untreated BRAFV600E dimer $\left(\mathrm{IC}_{50}=424 \mathrm{nM}\right)$ or the BRAFV600E monomer $\left(\mathrm{IC}_{50}=2760 \mathrm{nM}\right.$ ) (Fig. 6c, d). This enhanced potency for the second site of the $\mathrm{BRAF}^{\mathrm{V} 600 \mathrm{E}}$ dimer indicates positive co-operativity of binding to the second site induced by occupancy of the first site. In contrast, Vemurafenib showed very weak inhibition of both the second site within the BRAFV600E dimer $\left(\mathrm{IC}_{50}=2504 \mathrm{nM}\right)$ and the Encorafenib- 
Fig. 5 Targeting the allosteric site in BRAF with PHI1. Comparison of binding modes of PON (a) and PHI1 (b) with BRAFV600E in their corresponding crystal structures, illustrating interactions of PHI1 with the $\alpha \mathrm{C}$-allosteric site. Protein residues lining the base of $\alpha \mathrm{C}$-helix and HRD motif are shown in surface representation. Protein-ligand interface contact area (within $3.6 \AA$ of ligand atoms) is colored in orange (PON) and magenta (PHI1), respectively. $\mathrm{H}$-bonds are shown with dashed white lines. Protein parts are omitted for clarity. c Major PHI1-induced structural rearrangement of $\alpha \mathrm{C}$-helix (green) in $\mathrm{BRAF} 600 \mathrm{E} / \mathrm{PHI} 1$ complex compared to $\mathrm{BRAFV600E/PON} \mathrm{(orange).} \mathrm{The} \alpha \mathrm{C}$-allosteric site is shown in surface representation as in $\mathbf{b}$ and $\mathrm{PHI} 1$ atoms as van der Waals spheres. d Cartoon map showing molecular interactions between PHI1 and BRAFV600E. e Superposition of BRAFV600E/PHI1 (green) and BRAFV600E/PON (orange) complexes, demonstrates that PHI1 binding defines a new back pocket (BP-VI, red circle), according to KLIFS database nomenclature, which corresponds to the aC-allosteric site shown in $\mathbf{b}$. PON is classified as a BP-IV binder. The position of BP-V is also shown.

$\mathbf{f}$ KinomeEDGE ${ }^{\oplus}$ of PON and PHI 1 at $1 \mu \mathrm{M}$. Interaction maps of human kinases, including mutated, pathogen and lipid kinases, illustrating levels of PON and PHI1 binding (radii of red circles, see inset) giving better than $90 \%$ displacement of control binding (see "Methods" section). $\mathbf{g}$ Inhibition of kinase activity of $\mathrm{BRAF}^{\mathrm{V} 600 \mathrm{E}}, \mathrm{BRAFWT}$, and other tyrosine kinases targets, identified by KinomeEDGE ${ }^{\oplus}$, using SelectScreen (Invitrogen) in the presence of $100 \mu \mathrm{M}$ ATP. Half-maximal inhibition values $\left(\mathrm{IC}_{50} \pm \mathrm{SD}\right)$ of two technical replicates from $n=2$ independent experiments are tabulated. Source data are provided as a Source Data file.

untreated BRAFV600E dimer $\left(\mathrm{IC}_{50}=2425 \mathrm{nM}\right.$ ) (Supplementary Fig. 20), as expected by its negative allosteric effect. On the other hand, Ponatinib had similar inhibition activity $\left(\mathrm{IC}_{50}=495 \mathrm{nM}\right)$ for the second site compared to Encorafenib-untreated BRAFV600E $^{2}$ dimer $\left(\mathrm{IC}_{50}=452 \mathrm{nM}\right.$ ) (Supplementary Fig. 20). We excluded the possibility that positive co-opertivity arises from elimination of cellular activity from residual BRAFV600E monomers by Encorafenib pre-treatment, as monomeric species are not observed upon size-exclusion chromatography analysis of SKMEL239-C4 cell extracts (Supplementary Fig. 21). To confirm whether positive co-operativity by PHI1 is indeed unique property among BRAF aC-IN/type-II inhibitors, we compared second-site binding profiles of three inhibitors that inhibit BRAF monomers and dimers ${ }^{11,45}$. LY3009120 (Fig. 6e, f), AZ-628 (Fig. 6g, h), and TAK-632 (Fig. 6i, j) demonstrated very similar potency in inhibiting the second site within the BRAFV600E dimer or the Encorafenib-untreated $\mathrm{BRAF}^{\mathrm{V} 600 \mathrm{E}}$ dimer, suggesting equipotent binding of the first and second-site within the dimer by these inhibitors. In contrast, PHI1 exerts more potent inhibition of the second site of the BRAF ${ }^{\mathrm{V} 600 \mathrm{E}}$ dimer (Fig. 6k). To examine whether PHI's selectivity is linked to direct target engagement for BRAFV600E dimers, we performed Cellular Thermal Shift Assay (CETSA) in A375 and SKMEL239-C4 cells, under inhibitor-saturated conditions. CETSA showed that Ponatinib stabilizes BRAFV600E monomers in cells $\left(\Delta T_{\mathrm{m}}=\right.$ $\left.+6.3^{\circ} \mathrm{C}\right)$ more effectively compared to PHI1 $\left(\Delta T_{\mathrm{m}}=+3.4^{\circ} \mathrm{C}\right)$ (Supplementary Fig. 22a, b). In contrast, PHI1 and Ponatinib display similar stabilization effect with p61BRAFV600E dimers $\left(\Delta T_{\mathrm{m}}=+3.2^{\circ} \mathrm{C}\right) \quad$ (Supplementary Fig. 22c, d). Although $T_{\mathrm{m}}$ values may not reflect true binding affinity ranking under these conditions, these results are in agreement with the observed inhibition selectivity of PHI1 and Ponatinib (Fig. 6a, b).

Finally, we asked whether second-site positive co-operativity by PHI1 extents to RAS-dependent (class-II) or RAS-independent (class-III) non-V600E-mutated BRAF dimers and RASMUT/ BRAFWT dimers $^{46}$. To answer this, we assessed PHI1's inhibitory effect in lung adenocarcinoma H2087 (BRAF ${ }^{\mathrm{L} 597 \mathrm{~V}}$, class-II) and H1666 (BRAFG466V, class-III) cells, and SKMEL-2 and SKMEL-30 melanoma cell lines driven by RAS ${ }^{\mathrm{MUT}} / \mathrm{BRAF}^{\mathrm{WT}}$. In Encorafenibuntreated cells, we detected low p-ERK inhibition and various degrees of paradoxical activation by PHI1, which was pronounced in H1666 and BRAFWT melanoma cells (Fig. 7a, b). In contrast, after Encorafenib pre-treatment, PHI1 exhibited sub-micromolar p-ERK inhibition in both $\mathrm{H} 2087\left(\mathrm{IC}_{50}=485 \mathrm{nM}\right)$ and $\mathrm{H} 166\left(\mathrm{IC}_{50}=590\right.$ $\mathrm{nM}$ ) lung cancer cell lines, with more prominent effect in $\mathrm{H} 2087$, suggesting a strong positive co-operativity of PHI1 within nonV600E-mutated BRAF dimers present in these cells ${ }^{46}$ (Fig. 7a, b). However, PHI1 required low micromolar dose to inhibit signaling in $\mathrm{RAS}^{\mathrm{MUT}} / \mathrm{BRAFWT}$ without evidence of positive co-operativity upon Encorefenib pre-treatment (Fig. 7a, b). Furthermore, as with BRAFV600E dimers (Fig. 6i, j), aC-IN/type-II inhibitor TAK-632 demonstrated equipotent inhibition of BRAF dimer species and no positive co-operativity in these cell lines, while aC-OUT/type-I inhibitor Vemurafenib was largely inactive, without and with Encorefenib pre-treatment (Fig. 7e, f). Taken together, these results suggest that PHI1 has a unique specificity for the second-site of BRAF dimers and a distinct inhibition mechanism of positive cooperativity among different types of BRAF inhibitors (Fig. 8).

\section{Discussion}

BRAF mutational analyses in human cancer and complex biochemical mechanism of RAF activation intensified efforts for the development of selective and potent BRAF inhibitors $8,9,13,21$. Clinical BRAF inhibitors effectively target BRAFV600E monomers, but it is well established that such inhibitors do not inhibit oncogenic BRAF dimers and promote paradoxical activation in cells with wild-type BRAF ${ }^{11-14}$. Inhibitors that potently inhibit BRAF dimers may have a broader efficacy in several BRAF-dependent tumors and in combination with clinical BRAF inhibitors may also overcome adaptive and acquired resistance ${ }^{12,13,15,47}$. Recent drug development efforts have yielded aC-IN RAF inhibitors that equipotently inhibit BRAF monomers and dimers without selectivity ${ }^{1-14}$. In addition, a recent report identified an aC-OUT RAF inhibitor, PLX8394, which selectively disrupts BRAF dimers in RAS-independent BRAF-mutant-driven signaling ${ }^{45}$.

Here, we identified Ponatinb, an FDA-approved kinase inhibitor, as a hitherto uncharacterized potent BRAF inhibitor, which binds either BRAFV600E monomers or BRAF dimers. With either BRAF variant, Ponatinib promotes formation of inhibited BRAF/CRAF dimers and BRAF/MEK1/2 complexes. In various cellular contexts, the net inhibitory effects of Ponatinib will depend on the combined effect of its targeting for BRAF monomers and dimers and its simultaneous capacity to form new dimers ${ }^{12,13}$. Therefore, it is not surprising that Ponatinib is more effective in cells with activated BRAFV600E and low active RAS, as opposed to cells with active RAS ${ }^{\text {MUT- }}$ BRAFWT that can induce new RAF dimers due to RAF priming. Nevertheless, as it is suggested by the Ponatinib-induced BRAF dimer structure, Ponatinib has diminished negative allostery within dimers and inhibits each protomer (first site and second site) with the same potency. Recently developed aC-IN RAF inhibitors $^{11-14,45}$ (e.g., LY3009120), which have equipotent inhibition for BRAF monomers and dimers with similar efficacy to Ponatinib and promote new RAF dimers due to inhibitorinduced RAF priming, have entered clinical trials in oncogenic BRAF-dependent tumors such as melanoma, colorectal, and other advanced solid tumors. Therefore, our findings may have 
a
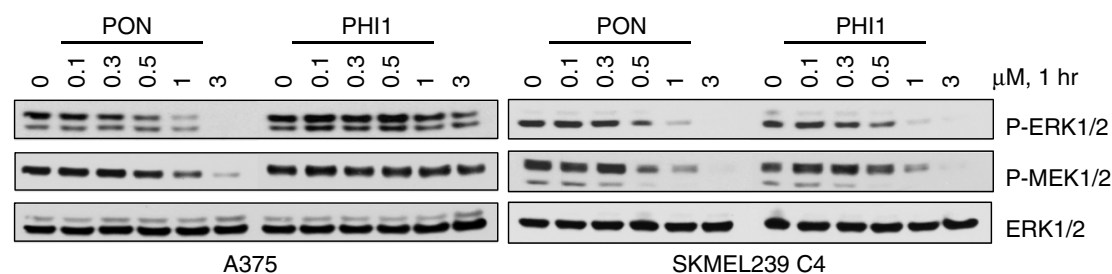

b
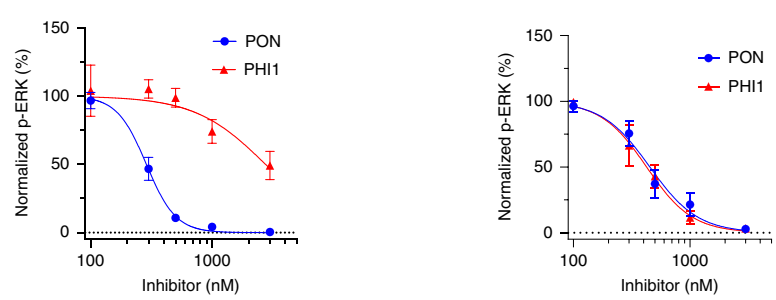

C

SKMEL239 C4
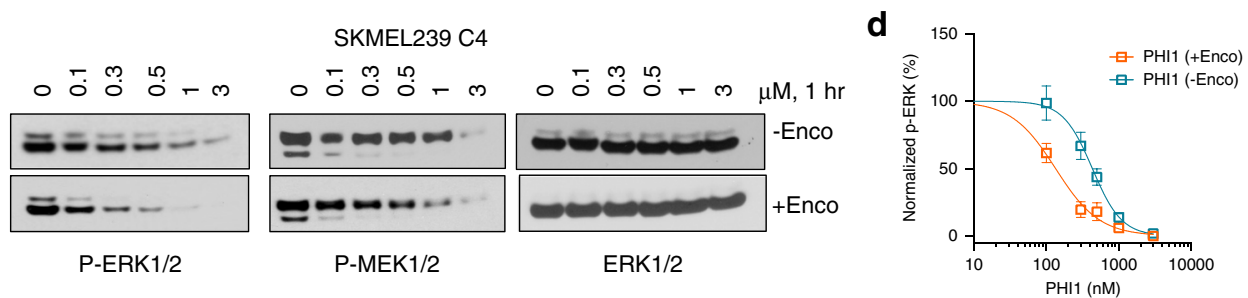

e

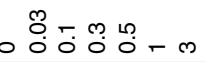

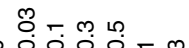

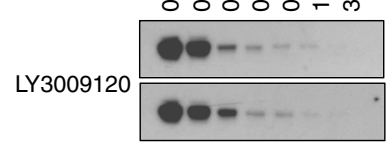

P-ERK1/2

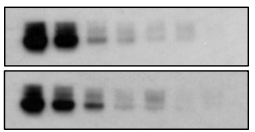

P-MEK1/2

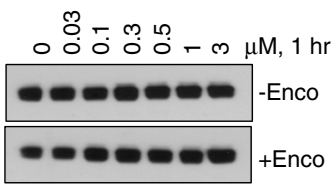

ERK $1 / 2$

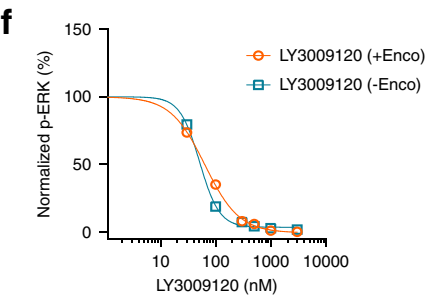

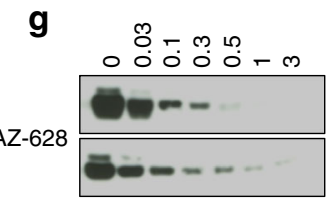

P-ERK1/2

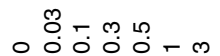

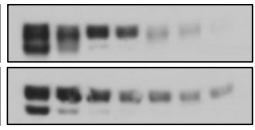

P-MEK1/2

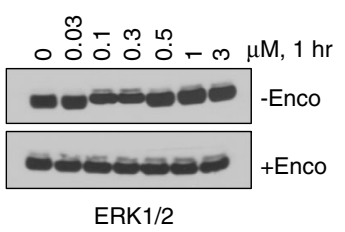

h

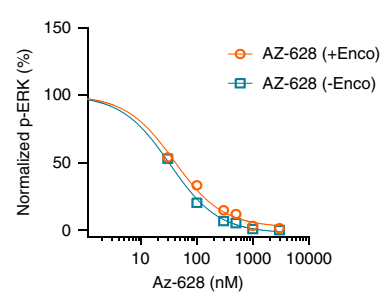

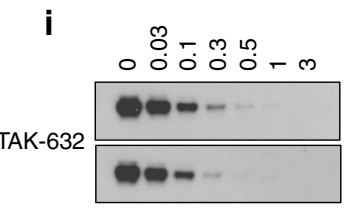

P-ERK1/2

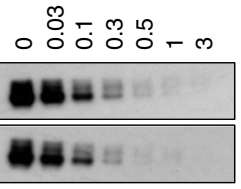

P-MEK1/2

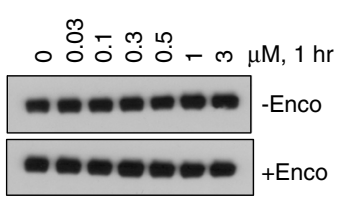

ERK1/2

k

SKMEL239 C4 IC50s of pERK inhibition (nM) Fold change

\begin{tabular}{|c|c|c|c|}
\hline & \multicolumn{2}{|c|}{ IC50s of pERK inhibition (nM) } & Fold change \\
\hline & -Enco & + Enco & \\
\hline PHI1 & $424 \pm 43$ & $134 \pm 14$ & -3.16 \\
\hline VEM & $2425 \pm 624$ & $2504 \pm 665$ & +1.03 \\
\hline PON & $452 \pm 45$ & $495 \pm 70$ & +1.09 \\
\hline AZ-628 & $33 \pm 3$ & $40 \pm 5$ & +1.21 \\
\hline LY3009120 & $51 \pm 2$ & $64 \pm 3$ & +1.25 \\
\hline TAK-632 & $83 \pm 9$ & $71 \pm 6$ & +1.16 \\
\hline
\end{tabular}

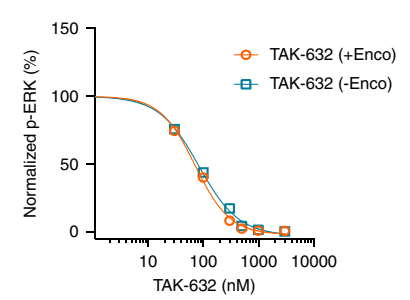

$$
\text { (1) }
$$

clinical implications and enable repurposing of Ponatinib in melanoma and other oncogenic BRAF-dependent tumors, more likely in a combination therapy with a MEK inhibitor or immunotherapy. The maximum plasma levels $(\sim 100 \mathrm{nM})$ and prolonged elimination half-life $(>24 \mathrm{~h})$ of Ponatinib in standard of care dose for CML suggest that Ponatinib would be clinically effective for targeting BRAF and ERK signaling inhibition ${ }^{48}$ Furthermore, potential dual targeting of BRAF and RTK inhibition by Ponatinib in a specific oncogenic context (e.g., $\mathrm{BRAF}^{\mathrm{V} 600 \mathrm{E}}$ and FGFR aberration) or in the context of adaptive resistance mechanisms through RTK signaling may be particularly effective to suppress ERK signaling. 
Fig. 6 PHI1 is a selective BRAF dimer inhibitor that displays positive co-operativity. a Melanoma A375 and SKMEL239-C4 cell lines were treated with increasing. concentrations of PON or PHI for $1 \mathrm{~h}$. Whole-cell lysate were assayed by western blot with the indicated antibodies to assess ERK-pathway inhibition. Representative blots from $n=3$ independent experiments are shown. $\mathbf{b}$ Quantitation of $p$-ERK inhibition; normalized values (mean \pm SEM, $n=3$ independent experiments) of p-ERK levels obtained by densitometry with corresponding fitted curves (see "Methods" section). c SKMEL239-C4 cells without Encorafenib ( - Enco) treatment and after Encorafenib (+Enco) treatment for $1 \mathrm{~h}$, followed by exchange with fresh medium for another hour, were treated with increasing concentrations of $\mathbf{c}$ PHI1, e LY3009120, g AZ-628 and i TAK-632 for $1 \mathrm{~h}$ and cell lysates were assayed by western blot with the indicated antibodies to assess ERK-pathway inhibition. A representative blot from $n=3(\mathbf{c}, \mathbf{d})$ and $n=2$ (e-j) independent experiments is shown. Normalized values and non-linear regression fits of p-ERK activity for different compounds in $\mathbf{c}, \mathbf{e}, \mathbf{g}$, and $\mathbf{i}$ is shown respectively. Error bars represent mean \pm SEM, $n=3$ (d) and mean of two replicates from $n=2$ independent experiments $(\mathbf{f}, \mathbf{h}, \mathbf{j})$. $\mathbf{k}$ Table summarizing $p$-ERK inhibition results from all inhibitors in Encorafenib-free and Encorafenib-treated SKMEL239-C4 cells. Source data are provided as a Source Data file.

a

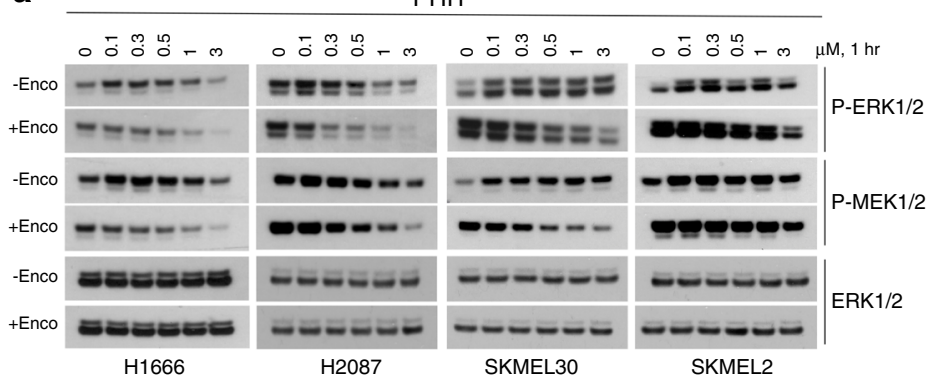

TAK-632

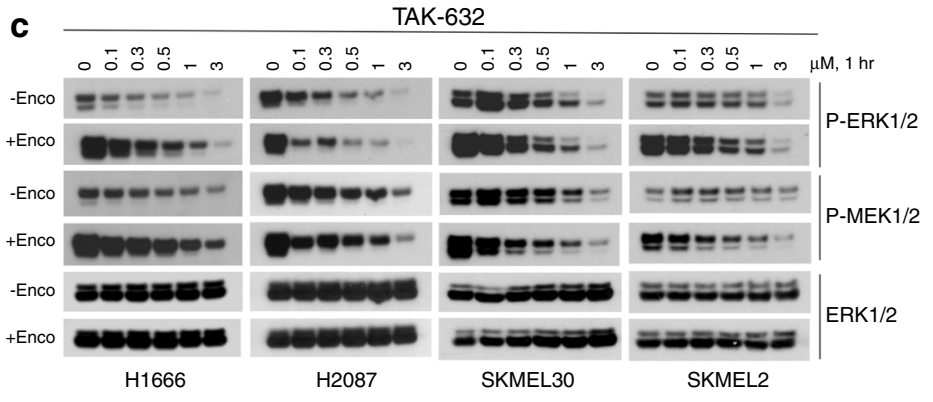

e

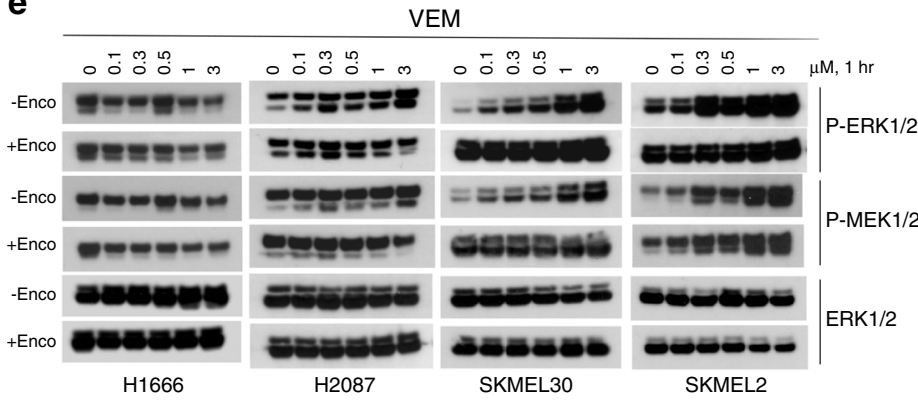

b
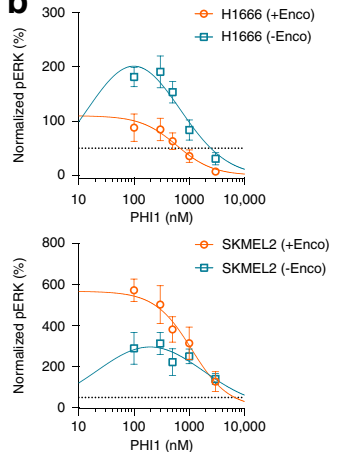

d 400
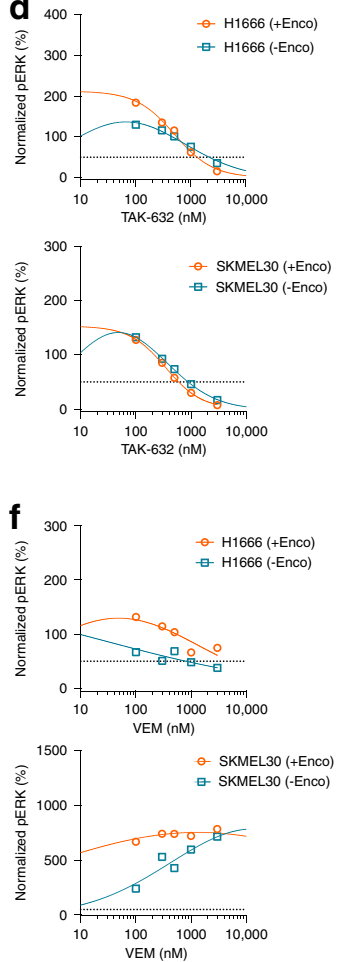
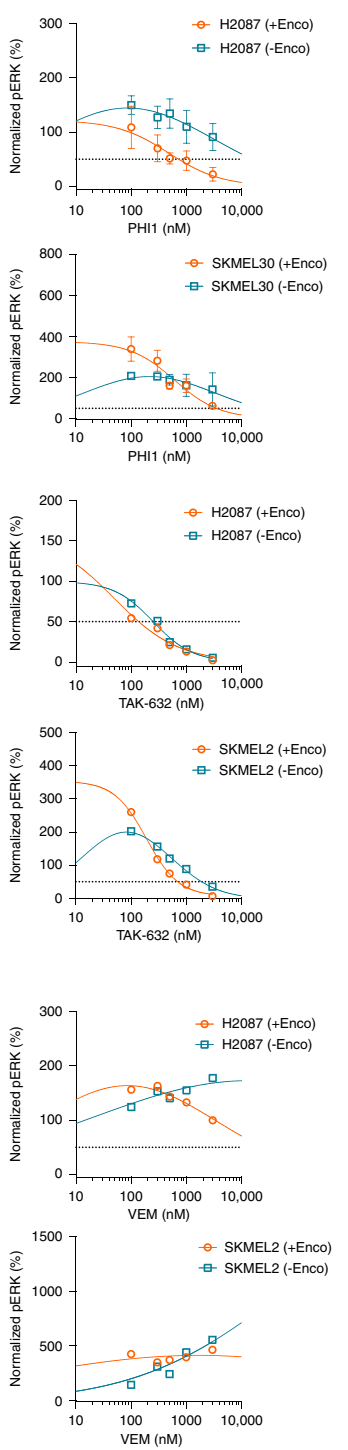

Fig. 7 Positive co-operativity in inhibition of non-BRAFV600E dimers by PHI1. Lung cancer H1666 (BRAFG466V), H2087 (BRAFL597V), and melanoma SKMEL-30 (BRAFWT/NRASQ61R), SKMEL-2 (BRAFWT/NRASQ61K) cells without Encorafenib (-Enco) treatment and after Encorafenib (+Enco) treatment for $1 \mathrm{~h}$, followed by exchange with fresh medium for another hour, were treated with increasing concentrations of a PHI1, c TAK-632, and d Vemurafenib for $1 \mathrm{~h}$ and cell lysates were assayed by western blot with the indicated antibodies to assess the ERK-pathway inhibition. A representative blot from $n=3$ (a, $\mathbf{b}$ ) and $n=2(\mathbf{c}, \mathbf{e})$ independent experiments is shown. Normalized values and non-linear regression fits of $p$-ERK activity for each treatment is shown in $\mathbf{b}, \mathbf{d}$, and $\mathbf{f}$, respectively. Error bars represent mean $\pm \mathrm{SEM}, n=3(\mathbf{b})$ and mean of two replicates from $n=2$ independent experiments (d, f). Notably, Encorafenib pre-treatment promotes paradoxical activation in these cells. Source data are provided as a Source Data file.

Our structural findings based on co-crystal structures of Ponatinib and PHI1 highlight that each inhibitor recognizes a distinct allosteric site and induces distinct dimers and aC-helix conformation. While Ponatinib promotes dimers with aC-
CENTRE conformation, PHI1 induces an aC-IN* conformation within the BRAFV600E dimer through interactions within its allosteric site. Indeed, we found that these interactions result in gain of specificity for PHI1 compared to Ponatinib for other 


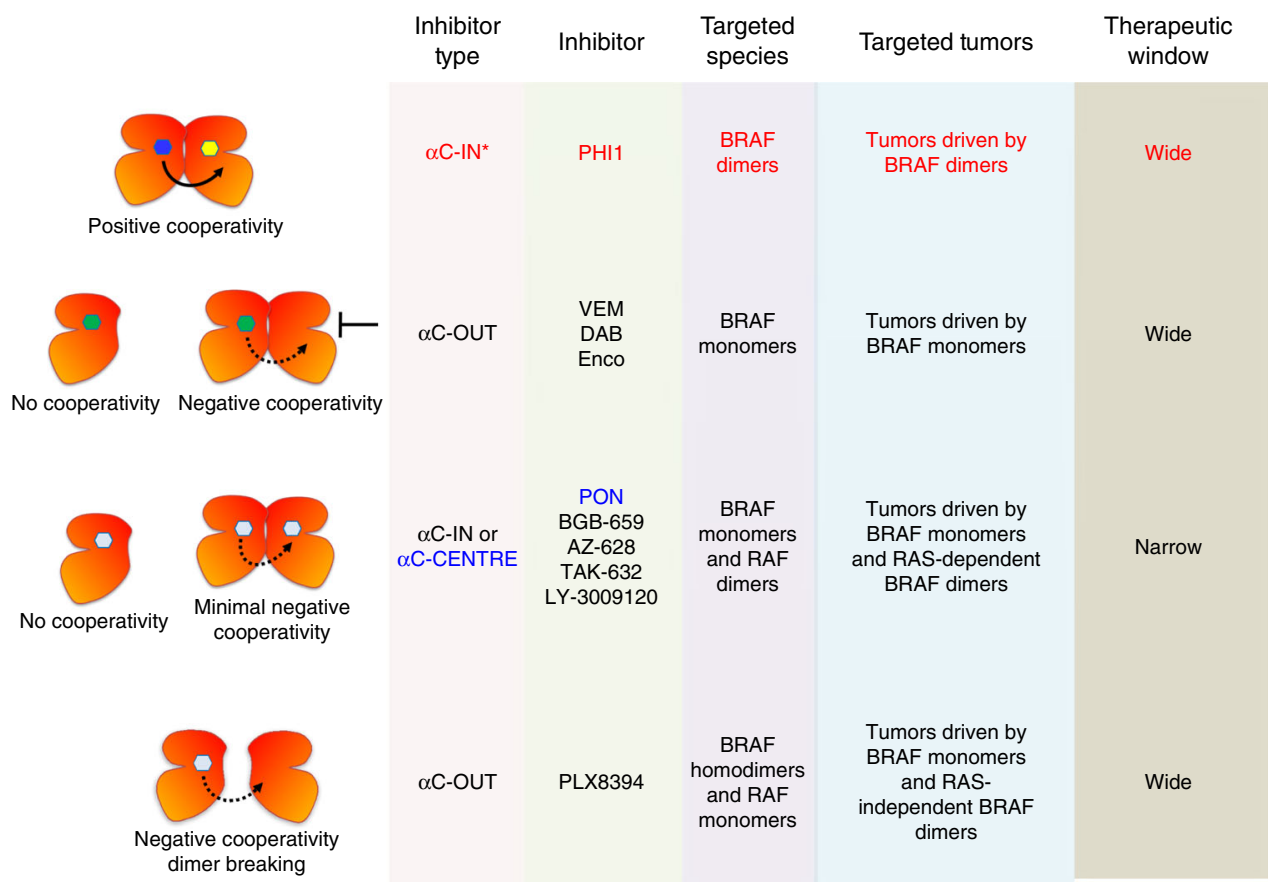

Fig. 8 Mechanisms of RAF inhibitors action. Scheme of four distinct mechanisms of RAF inhibitors action and representative RAF inhibitors for each mechanism. This work revealed that PHI1 displays a distinct structural, inhibitory, and therapeutic mode of action compared to previously characterized $\alpha \mathrm{C}$ IN and $\alpha$ C-OUT RAF inhibitors. Moreover, Ponatinib, an FDA-approved inhibitor, is an effective inhibitor of BRAF monomers and RAF dimers with a distinct structural binding mode.

kinase targets using the KinomeEDGE screen. Although this in vitro screen shows that a common subset of RTKs binds both Ponatinib and PHI1 (Supplementary Table S2), the distinct cellular BRAF monomer-dimer selectivity by the compounds is consistent with the effects of these compounds being RTKindependent. Remarkably, the $\mathrm{aC}$-allosteric site (BP-VI) revealed by PHI1 binding is not predicted in the current available kinase structures ${ }^{43}$. Additional drug design around this site should allow further fine-tuning of selectivity and potency for BRAF inhibition.

Our data demonstrate that PHI1 has a very weak inhibition for the $\mathrm{BRAF}^{\mathrm{V} 600 \mathrm{E}}$ monomers compared to the $\mathrm{BRAF}^{\mathrm{V} 600 \mathrm{E}}$ dimers, suggesting that interactions with the $\mathrm{aC}$-allosteric site (BP-VI) are favored in the dimer conformation while are unfavorable in the monomer conformation. Our data using the PHI2 compound showed that selectivity can be modulated by the head group attached to the Ponatinib and PHI1 core scaffold. Crystallization of PHI2 with BRAF or PHI1 with BRAF monomer was not successful, probably due to enhanced destabilization of aC-helix conformation. It is noteworthy that PHI1's distinct cellular selectivity compared to Ponatinib is not recapitulated by in vitro kinase inhibition systems (Supplementary Figs. 5 and 13), apparently due to absence of full structural and functional BRAF regulation ${ }^{13,14}$. We found that PHI1 inhibits more potently the second site of p61BRAF ${ }^{\mathrm{V} 600 \mathrm{E}}$ dimers when the first site within the dimer is occupied, uncovering the first paradigm of positive cooperativity for RAF inhibitors. This suggests that the allosteric coupling of PHI1 with aC-helix via specific interactions, promotes this positively co-operative effect, while Ponatinib, LY3009120, AZ-628, and TAK-632 target both sites with similar potency, as do other aC-IN/type-II BRAF inhibitors ${ }^{45}$.

PHIl also exhibited a co-operative mode of inhibition in oncogenic non-V600E BRAF dimers belonging to class-II and class-III, but not in RAS ${ }^{\mathrm{MUT}} \mathrm{BRAF}{ }^{\mathrm{WT}}$ driven melanomas. Although structural details on non-V600E mutants are elusive, these results demonstrate that both class-II and class-III oncogenic BRAF dimers species can be subjected to allosteric coupling by PHI1, driven by occupancy of the first protomer. Since signaling by class-III dimers depends mainly on heterodimerization with $\mathrm{BRAF} \mathrm{WT}$ or CRAF protomers ${ }^{46}$ these data indicate that activated RAF heterodimers are susceptible to this inhibitory mechanism. It is unclear why RAS-activated BRAFWT dimers are refractory to positive co-operativity by PHIl, but this may be related to enhanced priming and low occupancy of the first protomer.

In summary, RAF inhibitors can modulate BRAF inhibition by four distinct mechanisms (Fig. 8): (i) negative co-operativity by aC-OUT inhibitors that inhibit BRAF ${ }^{\mathrm{V} 600 \mathrm{E}}$ monomers (ii) no cooperativity by aC-IN or aC-CENTRE (Ponatinib) inhibitors that equipotently inhibit BRAF monomers and dimers (iii) dimer breaking activity such as aC-OUT PLX8394 inhibitor and a new category (iv) positive co-operativity by $\mathrm{aC}-\mathrm{IN}^{*}$ inhibitors exemplified by PHI1 that selectively inhibit $\mathrm{BRAF}^{\mathrm{V} 600 \mathrm{E}}$ and other oncogenic BRAF dimers. Inhibitors such as PHIl, may be particularly effective in blocking BRAF and ERK signaling in tumors expressing different BRAF dimer species of various classes, BRAF fusions or deletions ${ }^{45-50}$, when used alone or in combination with aC-OUT inhibitors that effectively inhibit the first site of the BRAF dimers.

To conclude, our strategy for identifying BRAF dimer kinase inhibitors demonstrates the significant value of screening specific phenotypic assays with additional kinase inhibitors to identify alternative uses and targets. There are several examples of kinase inhibitors that were developed for a specific kinase and indication, and later were found to have additional targets and effectiveness in a different cellular context or disease ${ }^{51-53}$. We foresee that a similar approach will be increasingly used to yield repurposing opportunities as well as chemical biology applications and drug development campaigns. Collectively, our data provide unexpected findings and useful mechanistic insights for BRAF targeting and hold promise for the development of nextgeneration inhibitors for the treatment of BRAF-dependent tumors. 


\section{Methods}

Compounds. Kinase inhibitors library was obtained from Selleck. Vemurafenib, Encorafenib, Trametinib, TAK-632, AZ-628, LY3009120, Lapatinib, and Ponatinib were purchased from Selleck and purity $>99 \%$ was confirmed by NMR and MS. PHI1 and PHI2 compounds were synthesized in $>99 \%$ purity and confirmed by NMR and MS. All compounds were dissolved in DMSO to a $10 \mathrm{mM}$ stock solution.

Antibodies. BRAF (1:1000, Santa Cruz sc-5284), BRAFV600E (1:1000, NewEast BioSciences 26039), CRAF (1:1000, Santa Cruz C-12), MEK1 (1:1000, Millipore Millipore, 07-641), MEK1/2 (1:1000, Cell Signaling 4694), P-MEK1/2 (1:1000, Cell Signaling 9154), ERK1/2 (1:1000, Cell Signaling 4696), P-ERK (1:200, Santa Cruz sc-7383), P-ERK1/2 (1:500, Cell Signaling 4370), ERK1 (1:200, Santa Cruz sc-94), Actin (1:10000, Invitrogen MA5-15739), GAPDH (1:5000, Sigma, G8795), IRDye800CW anti-mouse (1:800, LICOR 926-32210), and IRDye680RD antirabbit (1:800, LICOR 926-68071)

Cell culture. Cell lines were purchased from ATCC or provided by Poulikos Poulikakos laboratory. All cell lines were tested negative for mycoplasma contamination and authenticated by morphology and STR profiling. A375,

SKMEL239, SKMEL-30, and SKMEL-2 cells were grown in Dulbecco's modified Eagle's medium (DMEM) with 10\% fetal bovine serum (FBS), $1 \%$ Pen-Strep, and 1\% Glutamine. SKMEL239-C4 cells were grown in Dulbecco's modified Eagle's medium (DMEM) with $10 \%$ fetal bovine serum (FBS), $1 \%$ Pen-Strep, and $1 \%$ Glutamine in the presence of $1 \mu \mathrm{M}$ Vemurafenib. CALU6 cells were grown in Roswell Park Memorial Institute (RPMI) 1640 medium with 10\% FBS, 1\% PenStrep, and 1\% Glutamine. H1666 and H2087 cells were grown in RPMI 1640 medium with 5\% FBS, $1 \%$ Pen-Strep, and 1\% Glutamine.

In-cell-western screening. SKMEL239-C4 melanoma cells were plated in 96-well plate in DMEM 10\% FBS, $1 \%$ Pen-Strep, and $1 \%$ Glutamine, and allowed to seed overnight. Media was removed and replaced with fresh media containing $0.5 \mu \mathrm{M}$ Vemurafenib and treated with $5 \mu \mathrm{M}$ of corresponding kinase inhibitors and incubated for $3 \mathrm{~h}$. Cells were then fixed in $4 \%$ formaldehyde for $20 \mathrm{~min}$ at room temperature (RT) and washed four times with $0.1 \%$ Triton in $1 \times$ PBS for 5 min at RT with gentle rocking. Cells were then rinsed with $1 \times \mathrm{PBS}$ and stored in $1 \times \mathrm{PBS}$ at $4{ }^{\circ} \mathrm{C}$ for future in-cell-western (ICW). For ICW, we followed LiCor PI-140 0103 Doc \#988-07083 protocol with some modifications (https://www.licor.com/ documents/k12xs979o8ku50313v3r0n15n47w0f0c). In brief, cells were blocked with Odyssey blocking solution (LiCor) for $1 \mathrm{~h}$ at RT. Then cells were incubated with primary antibodies diluted in odyssey blocking buffer (1:200 P-ERK and 1:200 ERK1) for $2 \mathrm{~h}$ at RT, followed by four washes with $0.1 \%$ Tween- 20 in $1 \times$ PBS (PBST) for $5 \mathrm{~min}$ at RT. Then cells were incubated for $2 \mathrm{~h}$ at RT with secondary antibodies diluted in odyssey blocking buffer containing $0.2 \%$ Tween-20 (1:800 IRDye800CW anti-mouse and 1:800 IRDye680RD anti-rabbit) and washed 4 times with PBST for $5 \mathrm{~min}$ at RT. Cell were then rinsed once with $1 \times$ PBS, aspirated off and the plate was scanned with detection in both 700 and $800 \mathrm{~nm}$ channel using Odyssey Classic imager (ODY-0671). Quantification and analysis was performed using the Western Analysis tool from the Image Studio 3.1 software. Percent of phosphorylated-ERK1 was calculated as the total fluorescence levels of phosphorylated-ERK1 antibody staining divided by the total fluorescence levels of ERK1 antibody staining and normalized to percent of phosphorylated-ERK1 of untreated cells.

Analysis of ERK-signaling. Melanoma cells (A375, SKMEL239-C4, SKMEL-30, SKMEL-2) were plated in 6-well plates in DMEM with $10 \%$ FBS, $1 \%$ Pen-Strep, and $1 \%$ Glutamine to $70-80 \%$ confluency and lung cancer cells (H1666, H2087) to 90\% confluency in RPMI 1640 medium with 5\% FBS, $1 \%$ Pen-Strep, and 1\% Glutamine. SKBR3 cell were grown to $70 \%$ confluency in McCoy's 5 A Medium with $10 \%$ FBS, $1 \%$ Pen-Strep and $1 \%$ Glutamine and $2200 \mathrm{mg} / \mathrm{L}$ sodium bicarbonate. To assess ERK-signaling, cells were treated with DMSO or compounds for $1 \mathrm{~h}$ and analyzed by western blotting. For ${ }^{{ }^{2}}$ ncorafenib wash-out experiments, cells in 6-well plates were pre-treated with $1 \mu \mathrm{M}$ Encorafenib for $1 \mathrm{~h}$, washed $3 \times$ with PBS and incubated for $1 \mathrm{~h}$ with fresh medium, followed by DMSO or compound treatment. For evaluation of RTK-dependence of inhibition by Ponatinib, before Ponatinib treatment cells were pre-treated with $1 \mu \mathrm{M}$ Lapatinib for $1 \mathrm{~h}$ and washed $3 \times$ with PBS. Western blots were performed from whole-cell lysates (WCL) prepared in lysis buffer containing $50 \mathrm{mM}$ Tris- $\mathrm{HCl} \mathrm{pH} \mathrm{7.5,1 \%} \mathrm{NP40,150} \mathrm{mM} \mathrm{NaCl,}$ $1 \mathrm{mM}$ EDTA, and $10 \%$ glycerol in the presence of protease inhibitor cocktail (Roche). WCL were separated on 4-12\% NuPAGE MES gel (Invitrogen), transferred on a PVDF membrane, blocked for $1 \mathrm{~h}$, and immunoblotted with the corresponding antibodies. To increase data output, the multistrip western blotting method was used for a selection of experiments ${ }^{54}$.

Co-immunoprecipitation and kinase activity assays. Co-immunoprecipitation assays were performed from whole-cell lysate prepared in lysis buffer in the presence of protease inhibitor cocktail (Roche) and incubated at $4{ }^{\circ} \mathrm{C}$ overnight with gentle rotation, then protein-G beads were added and incubated for another $2 \mathrm{~h}$ at 4 ${ }^{\circ} \mathrm{C}$. Kinase activity assays were performed using BRAF Kinase Assay Kit (\#17-359), CRAF Kinase Assay Kit (\#17-360) and MAP Kinase/Erk Assay Kit (\#17-191), following protocols by the provider (Millipore) with some modifications. In brief, BRAF and CRAF, and MEK1 recombinant proteins were incubated for $15 \mathrm{~min}$ with inhibitors, then corresponding substrates were added and incubated for $30 \mathrm{~min}$ at $30^{\circ} \mathrm{C}$ and assayed via western blot.

Size-exclusion chromatography of SKMEL239-C4 cell extracts. SKMEL239-C4 melanoma cells were plated in 6-well plates in DMEM 10\% FBS, $1 \%$ Pen-Strep, and $1 \%$ Glutamine to $60 \%$ confluency. Cells were incubated in the absence of Vemurafenib (DMSO) or with $2 \mu \mathrm{M}$ Vemurafinib for $72 \mathrm{~h}$. After incubation cells were washed with PBS, collected and lysed in $50 \mathrm{mM}$ Tris- $\mathrm{HCl} \mathrm{pH} \mathrm{7.5,} 1 \% \mathrm{NP} 40$ $150 \mathrm{mM} \mathrm{NaCl}, 1 \mathrm{mM}$ EDTA plus protease inhibitors. Freshly lysed whole-cell lysates $(2.5 \mathrm{mg}$ total protein) were loaded onto a Superdex200 HR 10/300 (GE Healthcare) column and run at a flow rate of $0.2 \mathrm{~mL} / \mathrm{min}$. Fractions $(0.5 \mathrm{ml})$ were collected and western blotted for BRAFV600E.

p61BRAFV600E expression in HEK293H cells and PON treatment. HEK239H cells were seeded in 6-well plates $\left(1 \times 10^{6}\right.$ cells per well $)$ and transfected the following day using Lipofectamine 3000 (Invitrogen L3000008) according to the manufacturer's instructions. $2 \mu \mathrm{g}$ of p61BRAF ${ }^{\mathrm{V} 600 \mathrm{E}}$ plasmid (pcDNA-3 vector) DNA per well was employed. $24 \mathrm{~h}$ after transfection cells were treated with DMSO or increasing concentrations of Ponatinib for $1 \mathrm{~h}$. ERK signaling and BRAF V600E expression was assessed by western blot. Untransfected cells treated with DMSO or $1 \mu \mathrm{M}$ Ponatinib were used as negative control. In addition, HEK239H cells seeded in 6-well plates $\left(1 \times 10^{6}\right.$ cells per well) were transfected with Lipofectamine 3000 containing $2 \mu \mathrm{g}$ of p61BRAF ${ }^{\mathrm{V} 600 \mathrm{E}}$ plasmid or $2 \mu \mathrm{g}$ of p61BRAF ${ }^{\mathrm{V} 600 \mathrm{ER} 509 \mathrm{H}}$ plasmid (pcDNA-3 vector) DNA per well. $24 \mathrm{~h}$ after transfection cells were treated with DMSO or increasing concentrations of PHIl for $1 \mathrm{~h}$ and ERK signaling, and BRAF expression was assessed by western blot. Cells treated with DMSO or $10 \mu \mathrm{M}$ Vemurefenib were used as positive control for inhibition of p61BRAFV600E/R509H and no inhibition of $\mathrm{p} 61 \mathrm{BRAF}^{\mathrm{V} 600 \mathrm{E}}$ signaling.

Densitometric analysis and quantification. Densitometric data for P-ERK, P-MEK, ERK1/2 (MAPK cellular activity), or BRAF and GAPDH (CETSA analysis) from western blot scanned films were obtained using Image Studio software (LI-COR). Data were corrected to loading control (total ERK1/2 or GAPDH) and normalized to DMSO-treated bands (100\%) and blot backgrounds (0\%). IC $\mathrm{I}_{50}$ or $T_{\mathrm{m}}$ values were obtained from non-linear regression fits of normalized data to a fourparameter logistic curve (4PL), using GraphPad Prism 8.

3D culture of melanoma cells. 3D laminin-rich extracellular matrix cultures were prepared by trypsinization of melanoma cells from tissue culture plastic, seeding of single cells on top of a thin layer of Growth Factor-Reduced Matrigel (BD Biosciences, Franklin Lakes, NJ), and the addition of a medium containing 5\% Matrigel. The cells were seeded at the density of 40,000 cells per $\mathrm{cm}^{2}$. All 3D cell cultures were performed in DMEM supplemented with $5 \mu \mathrm{g} / \mathrm{mL}$ bovine insulin with Zinc (Gibco), $15 \mu \mathrm{g} / \mathrm{mL}$ bovine pituitary extract (Gibco), $0.5 \mathrm{ng} / \mathrm{mL}$ epidermal growth factor (Sigma-Aldrich, St. Louis, MO) and 2\% fetal bovine serum. 3D cultures were maintained with media and/or drug-treatment changes every second day, and photographs were taken at the end of the 5th day. The size of single cell colonies was estimated with ImageJ (Version 1.51).

Cellular thermal shift assay (CETSA) analysis. For CETSA analysis, cultured A375 or SKMEL239-C4 cells were washed with Dulbecco's phosphate buffered saline (DPBS) and split into $500 \mu \mathrm{L}$ aliquots (each containing 375 million cells) in the same buffer, containing $20 \mu \mathrm{M}$ (A375 cells) or $70 \mu \mathrm{M}$ (SKMEL239-C4 cells) of PON, PHI1 or DMSO. The samples were incubated for $1 \mathrm{~h}$ at room temperature, rotating. After compound incubation, samples $(50 \mu \mathrm{L}$ each) were transferred in PCR tubes and incubated for $3 \mathrm{~min}$ in a temperature gradient produced with a C1000 thermal cycler (Bio-Rad). Cells were immediately lysed by repeating freezethaw cycles $(3 \times$ times) in liquid nitrogen. Lysates were spun in a microcentrifuge at $15,000 \times g$ for $15 \mathrm{~min}$ at $4{ }^{\circ} \mathrm{C}$. Equal volumes of supernatants were run on 15 -well 4-12\% NuPAGE SDS-PAGE gels (Invitrogen), and analyzed by western blot. GAPDH, which is temperature insensitive under these conditions, served as loading control. Results were quantitated by densitometric analysis as described above.

Cloning, expression, and purification of BRAF. Human BRAF kinase domain (residues 443-723) with V600E mutation in addition to designed mutations to improve expression in E. coli 12 was cloned into the first multiple cloning site of a pET-28a vector, which expresses a hexa-histidine tag at the $\mathrm{N}$ terminus of BRAF. Recombinant protein was transformed and expressed into E. coli strain BL21Codon Plus (DE3)-RIPL (Agilent Technologies). Protein purification was performed by a rapid two-step procedure using nickel-affinity chromatography $(\mathrm{Ni}-$ NTA) followed by size-exclusion chromatography with Superdex200 HR 10/300 (GE Healthcare). Ponatinib or PHI1 at 1.5 molar excess to the protein sample were added immediately after elusion from Ni-NTA column. 
Crystallization. Purified complexes of $\mathrm{BRAF}^{\mathrm{V} 600 \mathrm{E}}$ kinase domain with Ponatinib or PHI1 were concentrated to $4.5-5.5 \mathrm{mg} / \mathrm{mL}$ using a filtration unit (Millipore). Initial crystallization hits of the complexes were obtained in the HT Crystal HT screen (Hampton Research) using the sitting-drop vapor-diffusion method and 96well Intelli-plates (Hampton Research) at $293 \mathrm{~K}$. Optimized crystals of BRAFV600E Ponatinib were generated in $2 \mathrm{M}$ ammonium sulfate and $5 \%(\mathrm{v} / \mathrm{v}) 2$-propanol and of $\mathrm{BRAF}^{\mathrm{V} 600 \mathrm{E}}$-PHI1 in $1 \mathrm{M}$ sodium citrate. Crystals were cryo-protected shortly in mother liquor supplemented with $20 \%(\mathrm{v} / \mathrm{v})$ ethylene glycol and flash-frozen in liquid nitrogen.

Structure determination. Diffraction data to $2.1 \AA$ resolution were collected using microbeam beamline 23-ID-D at Advanced Photon Source (APS) of Argonne National Laboratory. X-ray data were processed with the program iMosflm (Version 7.2.1 ${ }^{55}$. The $\mathrm{BRAF}^{\mathrm{V} 600 \mathrm{E}}$ structure bound to Ponatinib or PHI1 was solved by the molecular replacement method in Phaser of CCP4 suite (Version 7.0) ${ }^{56}$ using the BRAF/AZ-628 structure (PDB 4RZW) as search model. Structure refinement was performed using REFMAC (Version 5.8.0158) ${ }^{57}$. Statistics of the diffraction data and refinement are summarized in Supplementary Table S1. Coordinates have been deposited with the Protein Data Bank (BRAF ${ }^{V 600 E}-\mathrm{PON}$ complex: PDB 6P3D, BRAF ${ }^{\mathrm{V} 600 \mathrm{E}_{-} \mathrm{PHI}}$ complex: PDB 6P7G).

Structural analysis. Software was available through the SBGrid collaborative network ${ }^{58}$. Structural analysis and 3D-superpositions were performed in Pymol (Version 2.3, The PyMOL Molecular Graphics System, Schrödinger, LLC) and Maestro tools (Maestro, Schrödinger Release 2018-3, Schrödinger, LLC). Interface interaction analysis between ligands and $\mathrm{BRAF}^{\mathrm{V} 600 \mathrm{E}}$ was performed using PISA in CCP4 (Version 7.0).

Recombinant kinase activity assay. BRAF kinase assays were performed using the Z'-LYTE ${ }^{\text {mo }}$ enzymatic assay (Invitrogen, USA). Briefly, kinase activity was monitored in a cascade system consisting a mixture of inhibitor with BRAF or $\mathrm{BRAF}^{\mathrm{V} 600 \mathrm{E}} /$ inactive MAP2K1 (MEK1)/inactive MAPK1 (ERK2)/Ser/Thr peptide (Invitrogen) in $50 \mathrm{mM}$ HEPES $\mathrm{pH} 7.5,100 \mu \mathrm{M}$ ATP, $10 \mathrm{mM} \mathrm{MgCl} 2,1 \mathrm{mM}$ EGTA, and $0.01 \%$ Brij-35. Titrations were performed using a 1:3 dilution. Assays were performed using SelectScreen (Invitrogen).

Kinome specificity. The KINOMEscan ${ }^{\varpi}$ screening platform (Eurofins/DiscoverX) was used to assess binding specificity of Ponatinib and PHI1 at $1 \mu \mathrm{M}$ across a panel of diverse kinases (scanEDGE panel). Maps were generated using TREEspot software (Version 5.0, http://www.kinomescan.com) and display a circular representation of the kinase family tree based on kinase domain sequence. Selectivity score, a quantitative measure of compound selectivity, was calculated using the formula $S$-score $(10)=($ number of non-mutant kinases with $\% \mathrm{Ctrl}<10 \%) /($ number of non-mutant kinases tested), where \%Ctrl represents the percent of positive control compound that remain bound to each kinase in the presence of the test compound.

Pharmacophore-based drug design. An in silico library of 3D compounds based on eMolecules (www.emolecules.com) library of 6.5 million purchasable compounds was generated using LIGPREP (Schrödinger Release 2017-4, Schrödinger, LLC) and EPIK (Schrödinger Release 2017-4, Schrödinger, LLC). The in silico library contained approximately 13.8 million compounds with different ionization state at $\mathrm{pH} 7.0 \pm 2.0$, stereochemistry and tautomeric form, excluding potential Pan Assay Interference Compounds (PAINS) using PAINS definitions included in Canvas (Schrödinger Release 2017-4, Schrödinger, LLC). Conformation analysis of ligands was calculated using the OPLS3 force field. Phase (Schrödinger Release 2018-3, Schrödinger, LLC) module was used to generate a pharmacophore hypothesis and a $3 \mathrm{D}$ pharmacophore screen ${ }^{59}$. The coordinates of the BRAF/ Ponatinib structure were used and a pharmacophore hypothesis was generated to preserve trifluoro-phenyl and piperazine interactions of Ponatinib with BRAF and further additional interactions within the allosteric pocket. Pharmacophore hypothesis included six features as defined in Phase and included one aromatic ring, two positively charged groups, two hydrophobic points and one hydrogen bond acceptor. The pharmacophore screen searched the in silico library with the requirement to satisfy at least five out of the six pharmacophore features of the hypothesis. Two hundred hits were obtained that satisfied at least five out of seven pharamacophore constrains, comprising of diverse chemical groups. Fragments were computationally linked to the Ponatinib core structure (replacing the piperazine moiety) to produce Ponatinib hybrid inhibitors. Virtual compounds were prepared with LIGPREP and EPIK and docked to BRAFV600E using GLIDE (Schrödinger Release 2018-3, Schrödinger, LLC). Docked compounds that demonstrated excellent fit to the pharmacophore model and have the most favorable interaction energies were selected for synthesis. Physicochemical and AMDET properties including Lipinski rules, permeability, logP, metabolic liabilities, and hERG inhibition were evaluated using QikProp (Schrödinger Release 2018-3, Schrödinger, LLC) to maintain or improve Ponatinib properties.
Statistical analysis. The number of independent experiments for each data set is stipulated in the respective figure legend. Statistical significance for pair-wise comparison of groups was determined by two-tailed Student's $t$-test and by oneway ANOVA using GraphPad PRISM software (Version 7.0, GraphPad Inc., CA).

Reporting summary. Further information on research design is available in the Nature Research Reporting Summary linked to this article.

\section{Data availability}

Data generated or analyzed during the study are available from the corresponding authors upon reasonable request. $\mathrm{BRAF}^{\mathrm{V} 600 \mathrm{E}} / \mathrm{Ponatinib}$ and $\mathrm{BRAF}^{\mathrm{V} 600 \mathrm{E}} / \mathrm{PHI}$ structure coordinates have been deposited in the PDB with accession codes 6P3D and 6P7G, respectively. Source data are provided with this paper.

Received: 4 January 2020; Accepted: 5 August 2020; Published online: 01 September 2020

\section{References}

1. Lavoie, H. \& Therrien, M. Regulation of RAF protein kinases in ERK signalling. Nat. Rev. Mol. Cell Biol. 16, 281-298 (2015).

2. Kolch, W. Coordinating ERK/MAPK signalling through scaffolds and inhibitors. Nat. Rev. Mol. Cell Biol. 6, 827-837 (2005).

3. Vigil, D., Cherfils, J., Rossman, K. L. \& Der, C. J. Ras superfamily GEFs and GAPs: validated and tractable targets for cancer therapy? Nat. Rev. Cancer 10, 842-857 (2010).

4. Simanshu, D. K., Nissley, D. V. \& McCormick, F. RAS proteins and their regulators in human disease. Cell 170, 17-33 (2017).

5. Wellbrock, C., Karasarides, M. \& Marais, R. The RAF proteins take centre stage. Nat. Rev. Mol. Cell Biol. 5, 875-885 (2004).

6. Samatar, A. A. \& Poulikakos, P. I. Targeting RAS-ERK signalling in cancer: promises and challenges. Nat. Rev. Drug Discov. 13, 928-942 (2014).

7. Roberts, P. J. \& Der, C. J. Targeting the Raf-MEK-ERK mitogen-activated protein kinase cascade for the treatment of cancer. Oncogene 26, 3291-3310 (2007).

8. Davies, H. et al. Mutations of the BRAF gene in human cancer. Nature 417 949-954 (2002)

9. Wan, P. T. et al. Mechanism of activation of the RAF-ERK signaling pathway by oncogenic mutations of B-RAF. Cell 116, 855-867 (2004).

10. Freeman, A. K., Ritt, D. A. \& Morrison, D. K. The importance of Raf dimerization in cell signaling. Small GTPases 4, 180-185 (2013).

11. Yao, Z. et al. BRAF mutants evade ERK-dependent feedback by different mechanisms that determine their sensitivity to pharmacologic inhibition. Cancer Cell 28, 370-383 (2015)

12. Karoulia, Z. et al. An integrated model of RAF inhibitor action predicts inhibitor activity against oncogenic BRAF signaling. Cancer Cell 30, 485-498 (2016).

13. Karoulia, Z., Gavathiotis, E. \& Poulikakos, P. I. New perspectives for targeting RAF kinase in human cancer. Nat. Rev. Cancer 17, 676-691 (2017)

14. Agianian, B. \& Gavathiotis, E. Current insights of BRAF inhibitors in cancer. J. Med. Chem. 61, 5775-5793 (2018).

15. Sullivan, R. J. \& Flaherty, K. T. Resistance to BRAF-targeted therapy in melanoma. Eur. J. Cancer 49, 1297-1304 (2013).

16. Nazarian, R. et al. Melanomas acquire resistance to B-RAF(V600E) inhibition by RTK or N-RAS upregulation. Nature 468, 973-977 (2010).

17. Shi, H. et al. Melanoma whole-exome sequencing identifies (V600E)B-RAF amplification-mediated acquired B-RAF inhibitor resistance. Nat. Commun. 3 , 724 (2012).

18. Villanueva, J. et al. Acquired resistance to BRAF inhibitors mediated by a RAF kinase switch in melanoma can be overcome by cotargeting MEK and IGF-1R/ PI3K. Cancer Cell 18, 683-695 (2010).

19. Poulikakos, P. I. et al. RAF inhibitor resistance is mediated by dimerization of aberrantly spliced BRAF(V600E). Nature 480, 387-390 (2011).

20. Lito, P., Rosen, N. \& Solit, D. B. Tumor adaptation and resistance to RAF inhibitors. Nat. Med. 19, 1401-1409 (2013)

21. Holderfield, M., Deuker, M. M., McCormick, F. \& McMahon, M. Targeting RAF kinases for cancer therapy: BRAF-mutated melanoma and beyond. Nat Rev. Cancer 14, 455-467 (2014).

22. Zhang, C. et al. RAF inhibitors that evade paradoxical MAPK pathway activation. Nature 526, 583-586 (2015).

23. Bollag, G. et al. Vemurafenib: the first drug approved for BRAF-mutant cancer. Nat. Rev. Drug Discov. 11, 873-886 (2012).

24. Okaniwa, M. et al. Discovery of a selective kinase inhibitor (TAK-632) targeting pan-RAF inhibition: design, synthesis, and biological evaluation of 
C-7-substituted 1,3-benzothiazole derivatives. J. Med. Chem. 56, 6478-6494 (2013).

25. Peng, S. B. et al. Inhibition of RAF isoforms and active dimers by LY3009120 leads to anti-tumor activities in RAS or BRAF mutant cancers. Cancer Cell 28, 384-398 (2015).

26. Jin, T. et al. RAF inhibitors promote RAS-RAF interaction by allosterically disrupting RAF autoinhibition. Nat. Commun. 8, 1211 (2017).

27. Poulikakos, P. I., Zhang, C., Bollag, G., Shokat, K. M. \& Rosen, N. RAF inhibitors transactivate RAF dimers and ERK signalling in cells with wild-type BRAF. Nature 464, 427-430 (2010).

28. Hatzivassiliou, G. et al. RAF inhibitors prime wild-type RAF to activate the MAPK pathway and enhance growth. Nature 464, 431-435 (2010).

29. Heidorn, S. J. et al. Kinase-dead BRAF and oncogenic RAS cooperate to drive tumor progression through CRAF. Cell 140, 209-221 (2010).

30. Sullivan, R. J. et al. A phase I study of LY3009120, a Pan-RAF inhibitor, in patients with advanced or metastatic cancer. Mol. Cancer Ther. 19, 460-467 (2020).

31. Moerke, N. J. \& Hoffman, G. R. Development of in-cell western assays using far-red fluorophores. Curr. Protoc. Chem. Biol. 3, 39-52 (2011).

32. O'Hare, T. et al. AP24534, a pan-BCR-ABL inhibitor for chronic myeloid leukemia, potently inhibits the T315I mutant and overcomes mutation-based resistance. Cancer Cell 16, 401-412 (2009).

33. Huang, W. S. et al. Discovery of 3-[2-(imidazo[1,2-b]pyridazin-3-yl)ethynyl]4-methyl-N-\{4-[(4-methylpiperazin-1-yl)methyl]-3-(trifluoromethyl)phenyl\} benzamide (AP24534), a potent, orally active pan-inhibitor of breakpoint cluster region-abelson (BCR-ABL) kinase including the T315I gatekeeper mutant. J. Med. Chem. 53, 4701-4719 (2010).

34. Gozgit, J. M. et al. Potent activity of ponatinib (AP24534) in models of FLT3driven acute myeloid leukemia and other hematologic malignancies. Mol. Cancer Ther. 10, 1028-1035 (2011).

35. Gozgit, J. M. et al. Ponatinib (AP24534), a multitargeted pan-FGFR inhibitor with activity in multiple FGFR-amplified or mutated cancer models. Mol. Cancer Ther. 11, 690-699 (2012).

36. Lierman, E. et al. Ponatinib is active against imatinib-resistant mutants of FIP1L1-PDGFRA and KIT, and against FGFR1-derived fusion kinases. Leukemia 26, 1693-1695 (2012).

37. Tan, F. H., Putoczki, T. L., Stylli, S. S. \& Luwor, R. B. Ponatinib: a novel multityrosine kinase inhibitor against human malignancies. Onco Targets Ther. 12, 635-645 (2019).

38. Thevakumaran, N. et al. Crystal structure of a BRAF kinase domain monomer explains basis for allosteric regulation. Nat. Struct. Mol. Biol. 22, 37-43 (2015).

39. van Linden, O. P., Kooistra, A. J., Leurs, R., de Esch, I. J. \& de Graaf, C. KLIFS: a knowledge-based structural database to navigate kinase-ligand interaction space. J. Med. Chem. 57, 249-277 (2014).

40. Taylor, S. S., Keshwani, M. M., Steichen, J. M. \& Kornev, A. P. Evolution of the eukaryotic protein kinases as dynamic molecular switches. Philos. Trans. $R$. Soc. Lond. Ser. B Biol. Sci. 367, 2517-2528 (2012).

41. Haling, J. R. et al. Structure of the BRAF-MEK complex reveals a kinase activity independent role for BRAF in MAPK signaling. Cancer Cell 26, 402-413 (2014).

42. Giricz, O. et al. The RUNX1/IL-34/CSF-1R axis is an autocrinally regulated modulator of resistance to BRAF-V600E inhibition in melanoma. JCI Insight 3, e120422(2018)

43. Yueh C. et al. Kinase atlas: druggability analysis of potential allosteric sites in kinases. J. Med. Chem. 62, 6512-6524 (2019).

44. Davis, M. I. et al. Comprehensive analysis of kinase inhibitor selectivity. Nat. Biotechnol. 29, 1046-1051 (2011).

45. Yao, Z. et al. RAF inhibitor PLX8394 selectively disrupts BRAF dimers and RASindependent BRAF-mutant-driven signaling. Nat. Med. 25, 284-291 (2019).

46. Yao, Z. et al. Tumours with class 3 BRAF mutants are sensitive to the inhibition of activated RAS. Nature 548, 234-238 (2017).

47. Xue, J. Y. \& Lito, P. Quest for clinically effective RAF dimer inhibitors. J. Clin. Oncol. 38, 2197-2200 (2020).

48. Ye, Y.E., Woodward, C.N. \& Narasimhan, N.I. Absorption, metabolism, and excretion of [(14)C]ponatinib after a single oral dose in humans. Cancer Chemother Pharmacol. 79, 507-518 (2017).

49. Botton, T. et al. Genetic heterogeneity of BRAF fusion kinases in melanoma affects drug responses. Cell Rep. 29, 573-588 e577 (2019).

50. Zehir, A. et al. Mutational landscape of metastatic cancer revealed from prospective clinical sequencing of 10,000 patients. Nat. Med. 23, 703-713 (2017).

51. Pemovska, T. et al. Axitinib effectively inhibits BCR-ABL1(T315I) with a distinct binding conformation. Nature 519, 102-105 (2015).

52. Kuenzi, B. M. et al. Polypharmacology-based ceritinib repurposing using integrated functional proteomics. Nat. Chem. Biol. 13, 1222-1231 (2017).

53. Duong-Ly, K. C. et al. Kinase inhibitor profiling reveals unexpected opportunities to inhibit disease-associated mutant kinases. Cell Rep. 14, 772-781 (2016).
54. Kiyatkin, A. \& Aksamitiene, E. Multistrip western blotting to increase quantitative data output. Methods Mol. Biol. 536, 149-161 (2009).

55. Battye, T. G., Kontogiannis, L., Johnson, O., Powell, H. R. \& Leslie, A. G. iMOSFLM: a new graphical interface for diffraction-image processing with MOSFLM. Acta Crystallogr. Sect. D Biol. Crystallogr. 67, 271-281 (2011).

56. Winn, M. D. et al. Overview of the CCP4 suite and current developments. Acta Crystallogr. Sect. D Biol. Crystallogr. 67, 235-242 (2011).

57. Murshudov, G. N., Vagin, A. A. \& Dodson, E. J. Refinement of macromolecular structures by the maximum-likelihood method. Acta Crystallogr. Sect. D Biol. Crystallogr. 53, 240-255 (1997).

58. Morin, A. et al. Collaboration gets the most out of software. eLife 2, e01456 (2013).

59. Dixon, S. L. et al. PHASE: a new engine for pharmacophore perception, 3D QSAR model development, and 3D database screening: 1. Methodology and preliminary results. J. Comput. Aided Mol. Des. 20, 647-671 (2006).

\section{Acknowledgements}

We thank Jeffrey B. Bonanno, Tyler L. Grove, and staff of the X-ray Crystallography core facility of Albert Einstein College of Medicine for their support. Studies were supported by grants from the Melanoma Research Alliance and the Irma Hirschl Trust. Additional support from NIH grants R01CA238229, R01CA204314, and P30CA013330. X.C.R. was supported by an American Cancer Society fellowship (PF-16-097-CDD) and a T32 Training Grant T32 AG 23475. This research used resources of the Advanced Photon Source, a U.S. Department of Energy (DOE) Office of Science User Facility operated for the DOE Office of Science by Argonne National Laboratory under Contract No. DEAC02-06CH11357.

\section{Author contributions}

X.M.C-.R. designed and performed drug screening, biochemical, and cellular studies, B.A. designed, supervised and performed structural, drug design, biochemical, and cellular studies, N.G. and E.Z. performed cellular studies O.G., Y.Z., and A.V. designed and performed cellular studies. Y.W. performed protein production and crystallization studies. P.I.P. contributed to the research design and provided reagents. E.G. designed and supervised the study, and wrote the manuscript, which was edited by all authors.

\section{Competing interests}

E.G. and B.A. are inventors to US patent applications no. $62 / 810,056$ and no. $62 / 810,799$ filled by Albert Einstein College of Medicine that cover compounds and compositions to treat cancer related to this manuscript. E.G. has ownership interest in Stelexis Therapeutics, Selphagy Therapeutics, Aspida Therapeutics, and is a consultant/advisory board member for Stelexis Therapeutics, Selphagy Therapeutics, and Life Biosciences. None of the above companies have financial interest in this study or sponsored this research. No competing interests were disclosed by the other authors.

\section{Additional information}

Supplementary information is available for this paper at https://doi.org/10.1038/s41467020-18123-2.

Correspondence and requests for materials should be addressed to B.A. or E.G.

Peer review information Nature Communications thanks Donita Brady, Oliver Hantschel and the other, anonymous, reviewer(s) for their contribution to the peer review of this work. Peer reviewer reports are available.

Reprints and permission information is available at http://www.nature.com/reprints

Publisher's note Springer Nature remains neutral with regard to jurisdictional claims in published maps and institutional affiliations.

Open Access This article is licensed under a Creative Commons Attribution 4.0 International License, which permits use, sharing, adaptation, distribution and reproduction in any medium or format, as long as you give appropriate credit to the original author(s) and the source, provide a link to the Creative Commons license, and indicate if changes were made. The images or other third party material in this article are included in the article's Creative Commons license, unless indicated otherwise in a credit line to the material. If material is not included in the article's Creative Commons license and your intended use is not permitted by statutory regulation or exceeds the permitted use, you will need to obtain permission directly from the copyright holder. To view a copy of this license, visit http://creativecommons.org/ licenses/by/4.0/.

(C) The Author(s) 2020 